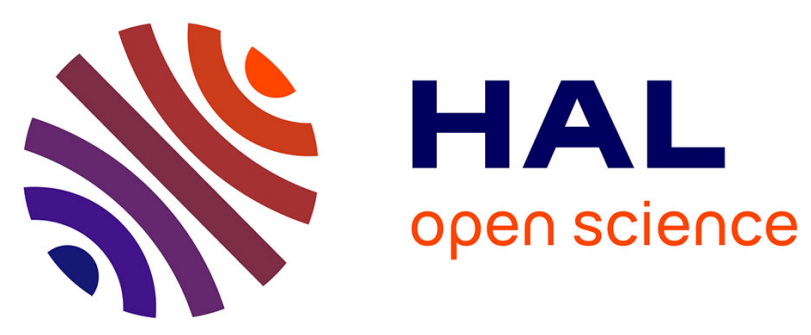

\title{
À la recherche de Goloupura
}

Christophe Pottier

\section{To cite this version:}

Christophe Pottier. À la recherche de Goloupura. Bulletin de l'Ecole française d'Extrême-Orient, 2000, 87 (1), pp.79-107. 10.3406/befeo.2000.3471 . halshs-02043537

\section{HAL Id: halshs-02043537 https://shs.hal.science/halshs-02043537}

Submitted on 21 Feb 2019

HAL is a multi-disciplinary open access archive for the deposit and dissemination of scientific research documents, whether they are published or not. The documents may come from teaching and research institutions in France or abroad, or from public or private research centers.
L'archive ouverte pluridisciplinaire HAL, est destinée au dépôt et à la diffusion de documents scientifiques de niveau recherche, publiés ou non, émanant des établissements d'enseignement et de recherche français ou étrangers, des laboratoires publics ou privés. 


\section{À la recherche de Goloupura}

In: Bulletin de l'Ecole française d'Extrême-Orient. Tome 87 N¹, 2000. pp. 79-107.

Citer ce document / Cite this document :

Pottier Christophe. À la recherche de Goloupura. In: Bulletin de l'Ecole française d'Extrême-Orient. Tome 87 N¹, 2000. pp. 79107.

doi : $10.3406 /$ befeo.2000.3471

http://www.persee.fr/web/revues/home/prescript/article/befeo_0336-1519_2000_num_87_1_3471 


\section{Abstract}

Christophe Pottier

In search of Goloupura

In this paper, the writer reconsiders an old hypothesis proposed in the 1930s by Victor Goloubew about the configuration of the first Angkorean capital built in the last years of the ninth century by king Yašovarman around his main temple on top of the Phnom Bakheng. Rarely and too briefly contested in the 1970s, this hypothesis -humorously named Goloupura- is still present and influences urban studies at Angkor, lending support to the idea of a square and geometric walled city, a shape supposed to be continuously applied for centuries, until Angkor Thorn.

The writer re-evaluates the findings of Goloubew's archaeological research and, using ground checkings, remote sensing, spatial analysis, epigraphic evidence and limited cores, he demonstrates that it is impossible to accept Goloubew's hypothesis. A fresh interpretation and a new dating are then proposed for the most important remains of Goloupura: what was previously considered to be the southwest part of the large moat surrounding the city appears to have been an ingenious waterway allowing a better link between the late twelfth-century capital with the lake Tonle Sap and the fluvial network. Finally, the writer concludes with a general view of the extension of Yašovarman's capital.

\section{Résumé}

Christophe Pottier

A la recherche de Goloupura

Cet article examine un aspect de l'hypothèse émise dans les années 1930 par Victor Goloubew à propos de la première capitale angkorienne, fondée à la fin du IXe siècle par Yašovarman autour du Phnom Bakheng. Alors que l'identification du temple établi au sommet de ce phnom avec le « templemontagne » de ce roi est désormais considérée comme une certitude, la restitution des limites de la cité - surnommée Goloupura - est nettement moins assurée même si elle a largement contribué - et contribue toujours - à propager l'image de la cité angkorienne entourée d'une enceinte carrée, assez semblable en fait à Angkor Thorn. Ce schéma idéal n'a été contesté que tardivement et brièvement par B. P. Groslier en 1979, mais reste encore fréquemment accepté, parfois légèrement modifié, et demeure ainsi très présent dans le paysage des études angkoriennes. L'article propose tout d'abord une réévaluation des arguments à l'origine de l'hypothèse de V. Goloubew. Puis les vestiges existants sont réétudiés suivant des approches complémentaires (analyse spatiale, télédétection, reconnaissances de terrain, examen de données épigraphiques et stratigraphiques) qui s'accordent à nier l'existence de Goloupura. Cette hypothèse devant être abandonnée, une autre interprétation et une nouvelle datation des vestiges monumentaux principaux sont alors proposées. Finalement, ces éléments permettent de brosser un tableau général de l'extension de la capitale de Yašovarman.

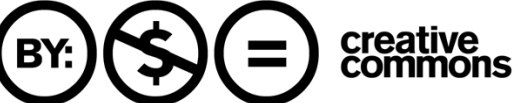




\title{
À la recherche de Goloupura '
}

\author{
Christophe POTTIER
}

Durant plus d'un siècle de patiente élaboration, la connaissance de la civilisation angkorienne s'est basée sur une somme d'interprétations successives, évidemment nourrie d'une part d'hypothèses qui se sont parfois effondrées au fil des années ou, plus heureuses, ont été confortées par de nouveaux éléments de compréhension. Toutefois, quelques hypothèses à s'être maintenues semblent essentiellement devoir leur longévité à l'absence tant de confirmation que de démenti. Elles se sont, en quelque sorte, "fossilisées ». C'est le cas, me semble-t-il, d'un aspect de l'hypothèse émise dans les années 1930 par Victor Goloubew à propos de la capitale fondée par Yaśovarman autour du Phnom Bakheng, "Yaśodharapura I». Si l'identification du Phnom Bakheng en tant que «templemontagne » ou «temple d'État» de Yaśovarman semble désormais une certitude, la restitution des limites de la cité de Yaśovarman est nettement moins assurée même si elle a souvent été entérinée depuis. Il s'agit pourtant d'un vaste quadrilatère centré sur le Phnom Bakheng et qui serait marqué par une enceinte d'environ $4 \mathrm{~km}$ de côté que Marchal surnomma amicalement "Goloupura " $^{2}$ - appellation que je conserverai ci-après pour désigner l'enceinte proposée par V. Goloubew autour de la ville de Yaśovarman.

Il n'en reste pas moins que le plan supposé de cette cité, considérée comme la première capitale à Angkor même, a très sensiblement participé à propager l'image de la cité angkorienne, rigoureusement conçue, entourée d'une enceinte carrée, renforçant ainsi l'idée d'un modèle géométrique délimité et axé, assez semblable en fait à Angkor Thom. Ce schéma idéal n'a été contesté que tardivement, par B. P. Groslier dans son article sur la «Cité hydraulique» $\left(1979: 174\right.$ et 182-183) ${ }^{3}$. Mais le vif débat déclenché par ce texte s'est focalisé sur ses conclusions, estompant du même coup l'intérêt de plusieurs passages dont l'un nie l'existence des limites de Yaśodharapura I proposées par V. Goloubew. Le texte de B. P. Groslier reste cependant trop bref pour avoir suffisamment ébranlé l'image idéale de l'urbanisme angkorien avant Angkor Thom. Même si depuis cet article quelques auteurs ont aussi abandonné Goloupura - sans pour autant justifier plus précisément cet

1. Le présent article emprunte un argumentaire déjà partiellement mis en œuvre dans le cadre de ma thèse de Doctorat (Pottier, 1999 : 164-167) ; il est cependant ici largement développé et complété par les résultats d'une récente série de carottages pilotes réalisés avec R. Fletcher dans le cadre du projet conjoint EFEO/Université de Sydney de recherches sur l'urbanisme médiéval en Asie du Sud-Est.

2. On trouve le terme "Goloupura » dans les journaux de fouilles de H. Marchal - par exemple : Journaux de fouilles de la Conservation d'Angkor $(J F) 11: 179-$, dans un contexte parfois ironique mais toujours sympathique qui témoigne de la bonne entente entre V. Goloubew et H. Marchal.

3. B. P. Groslier semble cependant avoir abondé dans le sens de Goloupura jusqu'au milieu des années 1960 (B. P. Groslier, 1961: 100 ; 1965 : 4). Son changement d'opinion pourrait être lié aux fouilles qu'il mena en 1966 dans les environs de Baksei Chamkrong, voir Rapports de la Conservation d'Angkor (RCA), 1966. 
abandon -, elle est encore fréquemment acceptée, parfois légèrement modifiée, et demeure très présente dans le paysage des études angkoriennes ${ }^{4}$.

Reconsidérer l'existence de Goloupura, quelque 70 ans après son apparition et plus de 20 après que B. P. Groslier l'a contestée, ne semble donc pas être un exercice dénué d'intérêt, tant ses répercussions restent actuelles. Ne serait-ce que parce que la détermination des limites et de la morphologie de Yaśodharapura I demeure un préalable pour dégager les caractéristiques de l'urbanisme angkorien et de l'occupation territoriale à cette époque. Capitale ouverte ou fermée dans une enceinte? La première solution offre la possibilité de reconsidérer dans une optique nouvelle les phénomènes de superposition mis en évidence à Angkor, tout comme elle permet de souligner la singularité de la composition d'Angkor Thom par rapport aux établissements urbains antérieurs.

Je m'attacherai donc dans les lignes qui suivent à apprécier l'hypothèse de V. Goloubew à la lumière de ses propres arguments et de l'analyse des vestiges existants en les replaçant dans leurs contextes spatial et chronologique. On verra ainsi que plusieurs approches complémentaires s'accordent à nier l'existence de Goloupura. Cette hypothèse devant être abandonnée, je proposerai une nouvelle interprétation de certains vestiges monumentaux et tenterai de brosser un tableau général de l'extension de la capitale de Yaśovarman.

\section{Les recherches de Victor Goloubew}

Durant les années 1920, les travaux de P. Stern et de G. Cœdès bouleversent la chronologie des monuments et suscitent de nouvelles problématiques de recherche. Entre autres, une lacune importante apparaît au $\mathrm{IX}^{\mathrm{C}}$ siècle pour la localisation de la "première " Yaśodharapura et du mont Śrī Yaśodharagiri (ou vnam kantāl) mentionnés dans la stèle de Sdok Kak Thom (Cœdès et Dupont, 1943-46:208, 224). P. Stern propose avec réserve le Phiméanakas pour remplacer le Bayon qui tenait précédemment ce rôle (1927:56 et 178181), mais cette solution n'est guère satisfaisante et $V$. Goloubew envisage dès 1930 d'identifier le vnam kantāl au Phnom Bakheng (1933a : 320). Cette identification se base non seulement sur le «style » du temple du Bakheng et sur son épigraphie ${ }^{5}$, mais aussi sur l'observation de la position du Phnom Bakheng par rapport à une double levée de terre située à environ $2 \mathrm{~km}$ au sud-ouest - nommée ci-après CP807 6 . V. Goloubew y voit les vestiges d'une vaste enceinte carrée, centrée sur le Bakheng, qui délimiterait l'emprise de la capitale de Yaśovarman (1933a : 322-323).

La vérification de cette identification fait alors l'objet de campagnes de prospections et de fouilles entre 1931 et 1937 , directement conduites sur le terrain par V. Goloubew avec la collaboration active de $\mathrm{H}$. Marchal ${ }^{7}$ et celle, plus ponctuelle, de G. Trouvé. Après un

4. Voir par exemple : Glaize, 1963 : 95 (sans modification dans la dernière édition de 1993 annotée par Boisselier) : Coedès, 1964a : 210 ; Boisselier, 1966:102-103; Jacques, 1978: 290-292; et, après 1979 : Higham, $1989: 326$; Jacques, $1990: 56-57$; Ishizawa \& Tamura, $1999: 165$.

5. Deux inscriptions découvertes avant les années 1930 (K. 464 et K. 558) précisent le nom ancien du temple du Bakheng : le Yaśodhareśvara (Cœdès, 1911 : 7 ; Finot, 1925 : 363-365 ; Jacques, 1970: 57-67).

6. Cette numérotation peu poétique ainsi que les numérotations utilisées plus bas dans cet article correspondent à celles que j'ai utilisées pour la réalisation d'une nouvelle cartographie de la région sud d'Angkor (Pottier, 1999).

7. H. Marchal partage l'identification du vnam kantāl, mais reste moins convaincu de l'existence de l'enceinte supposée de la ville. Il écrit dès 1931: "J'ai assez raillé Stern sur son deva-raja logé si à l'étroit dans la minuscule chapelle du Phimânakas, pour ne pas être pleinement satisfait du nouveau logement que lui attribue M. Goloubew. (...) Je me suis tout de suite rallié à la thèse de M. Goloubew, car l'abondance de ces vestiges, tous du même style, est une grande présomption en faveur de son 
séjour préliminaire en octobre 1931 durant lequel H. Marchal lui confirme la présence de sanctuaires de l'époque recherchée dans les environs du Phnom Bakheng, V. Goloubew revient à Angkor d'août à novembre 1932, puis de décembre 1933 à mars 1934 pour prospecter cette zone et y réaliser plusieurs sondages. La seconde mission s'attache aux abords immédiats du Phnom et aux environs du Bayon, à l'emplacement supposé de la partie septentrionale de Goloupura. Cette zone fait encore l'objet de deux dernières missions, d'avril à août 1936 et d'avril à juin 1937, qui mettent au jour de nombreux vestiges dans Angkor Thom, mais dont peu concourent finalement à asseoir l'hypothèse d'origine. Au contraire, ces dégagements aboutissent à l'élaboration d'une nouvelle hypothèse, celle d'une Yaśodharapura II datant de la première moitié du XI ${ }^{\mathfrak{c}}$ siècle, dont l'enceinte correspondrait approximativement à Angkor Thom ${ }^{8}$.

De nombreuses structures et quelques sanctuaires inédits sont relevés à l'occasion de ces travaux, essentiellement lors de prospections pédestres, car si les observations aćriennes - que V. Goloubew introduit à cette occasion à Angkor - soulignent dans les rares parties exemptes de forêt les caractéristiques principales de la composition urbaine présumée de Goloupura, elles fournissent surtout les axes majeurs selon lesquels sont entreprises les prospections au sol puis les fouilles ${ }^{9}$. Par ailleurs, la problématique urbaine qui guide les recherches de V. Goloubew le mène à innover en prenant en compte des vestiges même très réduits dont certains n'auraient - ou n'avaient - pas retenu l'attention précédemment. La notion de "vestige archéologique " s'élargit alors à des éléments non monumentaux, autres que des ruines de sanctuaires; par exemple, une attention toute particulic̀re est portée aux traces anciennes d'aménagements (digues, bassins, trapéang, terre-pleins...) et à leur localisation. Devant le nombre d'éléments repérés et l'importance d'en dresser une cartographie précise, il est fait appel à des topographes du Service géographique ${ }^{10}$.

Les recherches entreprises par V. Goloubew pour caractériser Yaśodharapura I présentent schématiquement deux volets dont seul le second se rapporte à Goloupura, qui

exactitude : j'ai justement à plusieurs reprises fait observer, que l'on trouvait un peu partout des vestiges de l'époque de Yaśovarman sauf à l'emplacement de la ville qu'on lui attribuait. La thèse de M. Goloubew fait disparaître cette anomalie " $(R C A$ 11/31). Par contre, on note chez lui un certain scepticisme face à la pauvreté des vestiges mis au jour que $V$. Goloubew interprète comme des preuves manifestes de l'existence de cette ville (par exemple: JF 9:57 à propos de l'angle sud-ouest de Goloupura CP784, ou JF 9: 31 et 33 pour les vestiges entre le Bayon et le Monument 486). Son scepticisme va croissant dès octobre 1932, au fur et à mesure que V. Goloubew lui « signale de nouveaux vestiges, très vagues, plus que vagues, mais que [Goloubew] incorpore à Yaśodharapura. (...) C'est tellement vague qu'avec de l'imagination, on peut tout voir !" (JF 9: 38-40). L'appellation de «Goloupura » semble d'ailleurs témoigner des doutes de H. Marchal.

8. «Mr. Goloubew considère que [ses] travaux révèlent l'existence, à l'intérieur d'Angkor Thom et à faible distance de la muraille qui entoure cette capitale, d'une enceinte complète, insoupçonnée jusqu'à présent (...). Pour des considérations d'ordre surtout technique, Mr. Goloubew incline à les attribuer à la première moitié du XI" siècle " (Trouvé, $R C A$ 05/36). Voir aussi Goloubew, 1936:622-623; 1937 : 651652. Cette hypothèse semble cependant avoir été moins suivie que Goloupura.

9. Goloubew, 1933a: $325 ; 1934: 578$. L'utilisation de prises de vues aériennes fera des émules et tant H. Parmentier que H. Marchal ou J. Lagisquet y ont recours dans les années suivantes, mais sans que des campagnes de terrain soient systématiquement entreprises pour vérifier les structures repérées.

10. M. Périnelli, adjudant du Service géographique de Hanoï, est mis à la disposition de l'EFEO en janvier 1934 pour dresser une carte au 1/10 000 des environs du Phnom Bakheng qui est achevée en mars (particllement reproduite à petite échelle dans Goloubew, 1934, fig. 17). Fin 1936, l'adjudant Hodemon réalise un relevé d'Angkor Thom au 1/10 000 (Marchal, 1937, pl. CX) et une cartographie de la région proche d'Angkor au 1/40 000 alors que M. Gassier, inspecteur des Travaux publics, établit le nivellement de nombreux éléments mis au jour (Marchal, 1937 : 656-664). 
nous intéresse particulièrement ici. Je rappellerai néanmoins que le premier, qui « fut déjà un grand progrès de notre connaissance d'Angkor ${ }^{11}$, concerne le Phnom Bakheng et son environnement immédiat : il aboutit à l'identification de ce phnom avec le vnam kantāl, mais aussi à la mise au jour d'un important aménagement d'ensemble au pied du phnom, contemporain de Yaśovarman (Goloubew, 1933a: 333-336; 1934 : 580-588), dont la connaissance a été depuis complétée par des recherches de B. P. Groslier ${ }^{12}$. Il y observe une composition assez similaire à celle du Bakong à Hariharālaya : une première douve « fut creusée au pied même de l'éminence volcanique. De ses axes médians s'élevaient les escaliers qui mènent au temple; là aboutissaient les chaussées le desservant (...). Un second fossé, plus large encore, la dédoublait à quelque $120 \mathrm{~m}$ à l'extérieur (...). Ces deux fossés enferment une couronne de templions » (B. P. Groslier, 1979 : 174).

Le second volet des travaux de V. Goloubew porte sur la limite de la cité de Yaśovarman, la Goloupura, et en particulier sur les éléments structurants de la composition urbaine - telle que V. Goloubew l'envisage -, c'est-à-dire les chaussées et, surtout, l'enceinte ${ }^{13}$. Ce volet découle directement de son hypothèse d'origine élaborée dès 1931, d'une ville centrée sur le Phnom Bakheng et délimitée par une enceinte vaste de plus de $16 \mathrm{~km}^{2}$. Au terme de sa seconde campagne, V. Goloubew souligne d'ailleurs avec une louable sincérité que, «bien avant que ne fût donné le premier coup de pioche, [il était] déjà à même de fixer sur un plan schématique les principaux éléments de la première Yaśodharāpura. Autrement dit, il s'agissait non pas de découvrir, dans le sens propre du mot, une ville inconnue, mais de contrôler sur un terrain encore peu exploré un ensemble de faits déterminés par ce raisonnement » (1934:600). Ce «contrôle » réalisé par le biais de prospections et de sondages renforce donc V. Goloubew dans son opinion. Elle peut être résumée ainsi ( $c f$. plan 1):

- L'enceinte de Goloupura est constituée à l'image des vestiges de levées de terre CP807 repérées au sud et au sud-ouest par les travaux cartographiques de Buat et Ducret en 1908, c'est-à-dire une douve large de $200 \mathrm{~m}$ encadrée par deux hautes et larges digues parallèles - que l'on nommera ici digue intérieure et digue extérieure.

- La chaussée médiane est-ouest d'Angkor Thom, la digue longeant la face orientale d'Angkor Vat et la berge orientale du baray occidental sont interprétées comme des vestiges de la digue intérieure de l'enceinte de Goloupura (1933a, pl. III ; 1934, pl. XIII).

- De même, la face sud de la levée de terre encadrant le trapéang Daun Méas (à l'ouest du Palais Royal) et longeant le Bapùon, ainsi qu'une digue est-ouest rejoignant les environs de l'angle nord-est du baray occidental, sont considérées comme les rares vestiges de la digue extérieure de Goloupura (1933a : 336-340).

- Du Phnom Bakheng rayonnent quatre chaussées axiales dont il est envisagé que leurs extrémités rejoignaient au moins la digue intérieure de Goloupura.

11. B. P. Groslier, $1979: 174$.

12. B. P. Groslier entreprend en 1966 un programme de restauration et de mise en valeur des sanctuaires au pied du Phnom Bakheng «destiné (...) à redessiner les vrais dispositifs marquant la Cité du Bakheng " ( $R C A$ 1970). À la fermeture des chantiers en 1972, les Prasat Bei 412, Thmar Bay Kaek 411, Bay Kaek GT6, Sok Krô Op CP491 et Rong Lmong GT3 avaient été restaurés.

13. V. Goloubew se donne un programme en cinq points pour ses recherches en 1932 : trois points concernent à des degrés divers - l'emplacement présumé de l'enceinte de la ville (1933a: 326). «En fait d'éléments caractéristiques d'une cité, je n'entrevoyais, en somme, qu'un réseau d'avenues rectilignes, avec quatre grandes artères correspondant aux quatre orientations cardinales et partant du pied de la colline dominée par le temple du Devarāja » (1933a : 323). 


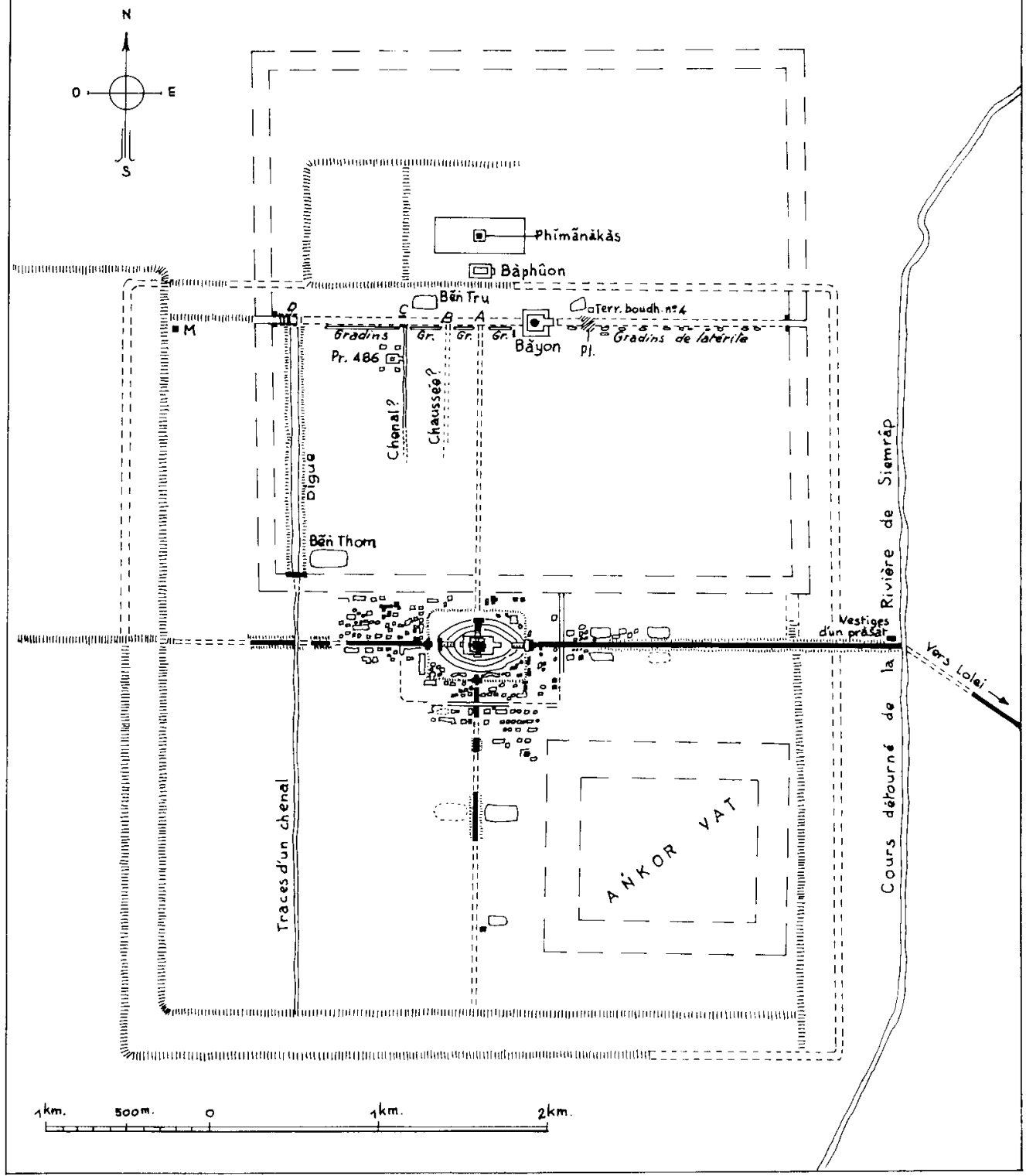

Plan 1 - Plan de la ville de Yaśovarman (Arikor I)

d'après les travaux de V. Goloubew et H. Marchal (décembre 1933 - mars 1934). 


\section{Une logique d'assemblage tardive}

Ce schéma entraîne une première série de commentaires " généraux » sur l'organisation et l'assemblage des éléments constitutifs de cette Goloupura, et sur le contexte dans lequel elle s'inscrit. Soulignons en préambule une réflexion générale sur le plan de Goloupura: dès l'origine, V. Goloubew avait envisagé le Bakheng comme le centre géométrique du tracé de la ville. Mais la recherche d'éléments susceptibles de correspondre aux vestiges de Goloupura l'a entraîné finalement à admettre un important décentrement du Bakheng (cf. plan 1). Pour justifier ce qu'il qualifie d' 'anomalie », il s'appuie sur l'opinion de $\mathrm{H}$. Parmentier qui considère certains désaxements francs des enceintes comme coutumiers dans les plans d'aménagements angkoriens. Si cette remarque de $\mathrm{H}$. Parmentier est parfaitement justifiée pour de nombreux cas de temples, elle demeure moins évidente en ce qui concerne les aménagements "urbains»: les exemples de villes angkoriennes à enceinte extérieure sur lesquels se baser restent en effet limités ${ }^{14}$. Il n'en demeure pas moins que, même si on devait retenir l'opinion de H. Parmentier, la centralité d'origine du Bakheng ressort malmenée dans le schéma final tellement ce dernier est adapté aux quelques vestiges pouvant évoquer la double levée de terre et la douve de Goloupura.

D'autre part, les deux digues de CP807 à l'origine de l'hypothèse de V. Goloubew se présentent dans un contexte dont la logique semble liée à des ouvrages nettement postérieurs à l'époque du Bakheng. B. P. Groslier avait déjà souligné cet aspect pour proposer une date plus tardive pour la double levée de terre CP807 (1979: 182). Bien que l'explication donnée par B. P. Groslier pour la fonction de CP807 ne paraisse pas entièrement convaincante ( $c$. infria), cette catégorie d'arguments à opposer à Goloupura doit être retenue. La situation relative des divers ouvrages les uns par rapport aux autres (cf. plan 3) et la cohérence - voire la logique - spatiale qui en résulte permettent en effet de mettre en évidence des relations de chronologies relatives. À l'est, les digues de CP807 s'achèvent contre la digue nord-sud qui longe la douve orientale d'Angkor Vat et qui se poursuit sur $150 \mathrm{~m}$ plus au sud avant de se retourner vers l'est pour franchir en chicane la rivière de Siemreap et se prolonger vers l'est (CP806) ${ }^{15}$. Cet arrêt abrupt de " l'enceinte " CP807 au droit de la digue nord-sud qui longe Angkor Vat suggère déjà que la première est au moins contemporaine de la seconde, et donc probablement du temple lui-même si l'on envisage que cette digue lui est justement liée. À l'autre extrémité, à l'ouest, «l'enceinte » CP807 se raccorde à l'angle sud-est du baray oceidental. Les prises de vues aériennes ne montrent aucune trace d'une éventuelle prolongation de la digue extérieure dans le périmètre du baray, à sec dans cette zone, et il est donc très improbable que la digue extérieure y ait jamais existé. Par ailleurs, bien que la digue orientale du baray et celle intérieure de CP807 se rejoignent, elles ne présentent ni la même section ${ }^{16}$ ni exactement la même orientation. Les prises de vues aériennes et les couvertures topographiques récentes montrent en effet une différence légère mais sensible (supérieure à $1^{\circ}$ ) dans les alignements, laissant penser que ces deux ouvrages ne proviennent pas d'un même

14. Hormis Angkor Thom, citons, par exemple, les cas du Prah Khan de Kompong Svay et Pimay. L'exemple du Bakong ne peut être retenu ici : Goloupura constitue une enceinte supplémentaire qui n'existe pas à Bakong.

15. Ces observations issues des prospections coïncident avec les levés topographiques de l'IGN et JICA. La configuration de cette zone a été, depuis la carte de Buat et Ducret, sujette à plusieurs interprétations divergentes et erreurs cartographiques. Voir par exemple : Parmentier, 1933: 1116.

16. Cet argument peut néanmoins s'expliquer par le surhaussement postérieur de la digue du baray. 
projet. La configuration de cette zone suggère que CP807 s'est raccordé sur l'angle du baray, et qu'il est donc postérieur à ce dernier ${ }^{17}$. Un premier examen du contexte dans lequel s'inscrit CP807, l'angle le plus visible de Goloupura, tend donc à nier l'existence de celle-ci ici en attribuant à la réalisation de CP807 une date nettement postérieure au début du $\mathrm{X}^{\mathrm{c}}$ siècle.

\section{L'absence de vestiges}

Un second examen plus détaillé est nécessaire pour évaluer l'existence de Goloupura : il s'agit de l'analyse des vestiges repérables le long du tracé supposé de la ville de Yaśovarman. Force est de constater une certaine pauvreté des vestiges mis au jour, voire même souvent l'absence d'éléments véritablement probants. Cela ressort d'ailleurs directement du journal de $\mathrm{H}$. Marchal alors même qu'il suivait étroitement les recherches de V. Goloubew ${ }^{18}$. Cette «absence» ou cette "faiblesse» a amené certains auteurs à proposer plusieurs amendements au schéma primitif, mais sans toutefois remettre en cause l'hypothèse de V. Goloubew. On a ainsi suggéré que des tronçons importants de cette enceinte n'avaient jamais été achevés, voire même commencés (Boisselier, 1966 : 102103), ou que l'enceinte n'était composée que d'une seule digue - la digue intérieure - et d'une douve (Jacques, 1978: 291). Cette pauvreté de vestiges et leur absence sur les parties restituées par V. Goloubew constituent naturellement un argument de choix que B. P. Groslier n'a pas manqué d'avancer pour contester l'ensemble de Goloupura (1979: 182). Il reste que l'examen détaillé de ces vestiges n'a été ni développé ni même repris depuis les années 1930.

Malgré son côté parfois fastidieux, nous entreprendrons ici cet examen du tracé de Goloupura, dans le sens trigonométrique à partir d'Angkor Thom, en couplant les informations «brutes » recueillies par V. Goloubew et $\mathrm{H}$. Marchal avec nos analyses des prises de vues aériennes ${ }^{19}$ et des cartographies récentes ${ }^{20}$, complétées par certaines observations issues de nos reconnaissances de terrain ${ }^{21}$.

17. Cette chronologie a aussi été soulignée par B. P. Groslier (1979: 183). Cf. infra.

18. Par exemple, le $1^{\text {cr }}$ novembre $1932, \mathrm{H}$. Marchal note dans son journal lors de recherches à l'ouest du Bayon: "Comment prouver [à V. Goloubew] que quelques briques ou morceaux de latérite épars sur un vague tumulus n'ont pas été jadis - entre les années 889 et 910 - des fondations du roi Yaśovarman? Je n'ai aucun argument valable : les poteries ou tessons découverts par lui dans les fouilles qu'il a fait exécuter lui prouvent l'époque de Yaśovarman et il les recueille avec soins. Elles sont du type courant rencontré par moi depuis que je fais des fouilles dans le parc d'Angkor ou ailleurs ! ( $(J F 10$ : 88).

19. On a principalement utilisé ici une couverture stéréoscopique au 1/25000 réalisée par Finmap en février 1992, associée au besoin aux prises de vues aériennes plus anciennes : une couverture de l'IGN en 1953-1954 au 1/40 000 et une de Williams-Hunt de 1945-1946 au 1/47 600 environ.

20. La première couverture topographique au $1 / 10000$ a été réalisée à l'instigation de B. P. Groslier au début des années 1960 par l'IGN et l'EFEO (publiée dans Dumarçay \& Pottier, 1993, pl. 2-26). Récemment, JICA a réalisé une couverture topographique au 1/5000 et au 1/10 000 (JICA, 1998).

21. Hormis la zone couverte par Angkor Thom, nous avons parcouru la majorité du tracé de Goloupura. 


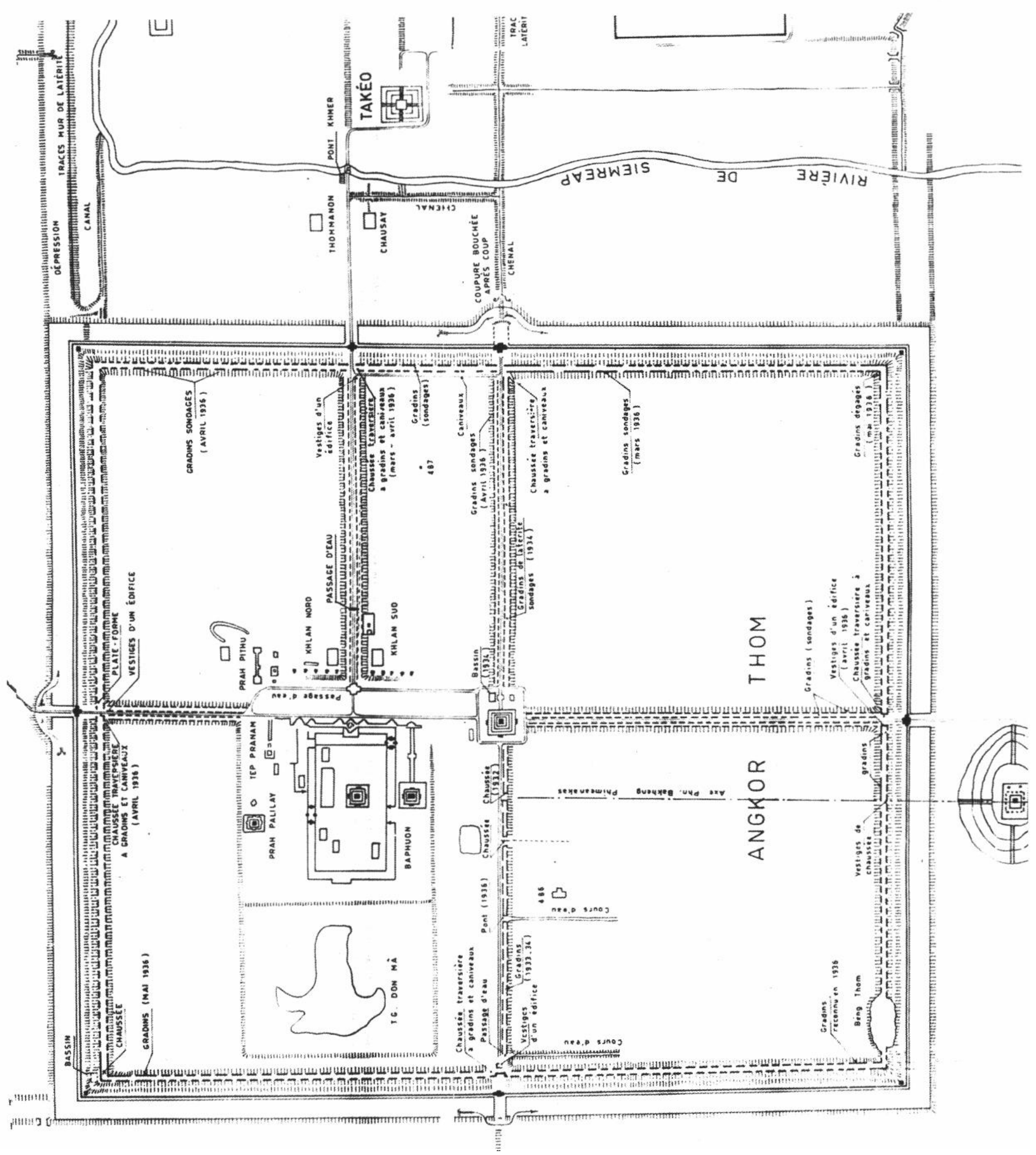

Plan 2 - Plan d'Angkor Thom montrant les fouilles de M. Goloubew en 1932-1933-1934-1936. 


\section{Face nord}

Dans Angkor Thom, les éléments repérés par V. Goloubew (cf. plan 2) ${ }^{22}$ consistent principalement en des chaussées et des canaux souvent parementés de latérite. Difficilement datables par eux-mêmes sans données complémentaires, ces vestiges montrent une organisation qui semble devoir être rattachée à celle de la capitale de Jayavarman VII plutôt qu'à une éventuelle capitale antérieure du $\mathrm{XI}^{\mathrm{c}}$, encore moins du $\mathrm{X}^{\mathrm{c}}$ siècle. Aussi, considérer à l'instar de V. Goloubew (1933a : 336-337) l'actuelle chaussée dans l'axe estouest d'Angkor Thom comme un vestige de la digue intérieure de Goloupura impose de négliger de nombreuses différences entre ces deux structures linéaires, ne serait-ce que la différence de largeur, du simple au triple ${ }^{23}$. De même, il apparaît probable que les vestiges découverts au croisement ${ }^{24}$ de l'axe Bakheng - Phiméanakas et de la chaussée ouest du Bayon, interprétés par V. Goloubew comme un indice «de tout premier ordre " pour l'existence d'une chaussée primitive nord de Goloupura (1933a : 332-333; $1934: 588$ et fig. 12), ne sont rien d'autre qu'un élément particulier - mais non unique ${ }^{25}$ - du canal parementé qui borde la face sud de la chaussée du Bayon. Enfin, il est difficile de suivre l'interprétation de V. Goloubew qui voit une trace de la digue extérieure de Goloupura dans la digue qui longe la face méridionale du Bapùon: tous les auteurs, y compris $\mathrm{V}$. Goloubew, la considèrent comme postérieure à ce temple et aucun état antérieur n'y a jamais été repéré $(1933 \mathrm{a}: 338)^{26}$. L'examen des recherches menées par V. Goloubew permet donc de constater, à la suite de B. P. Groslier, que les nombreux éléments mis au jour n'apportent finalement aucune confirmation de l'existence du tracé septentrional de Goloupura.

Près de l'angle nord-est du baray occidental, V. Goloubew avait reconnu l'extension de la chaussée d'Angkor Thom jusqu'au baray et, sous la broussaille, un tronçon de digue orienté est-ouest pouvant correspondre à un vestige de la digue extérieure de Goloupura (Goloubew, 1933a : $339 ; J F$ 9:62). Cette zone étant nettement plus dégagée actuellement, il est aisé d'y constater que ce tronçon existe bien, mais seulement sur une longueur de $300 \mathrm{~m}$ à partir de la digue du baray, pour se retourner vers le nord et rejoindre $200 \mathrm{~m}$ plus haut une autre digue est-ouest bordée par un chenal au nord. Les prises de vues aériennes et les cartographies topographiques récentes montrent aussi que le tronçon suit une direction qui n'est en rien parallèle à la chaussée d'Angkor Thom. Cet ensemble d'endiguements forme ainsi ce qui a pu être une pièce d'eau de $300 \times 200 \mathrm{~m}$, désormais

22. Les divers éléments mis au jour par V. Goloubew sont repérés sur la carte au 1/10 000 de 1936 (Marchal, 1937, pl. CX). Le plan 2 reproduit ci-contre a été réalisé en 1963, sans doute sous la direction de B. P. Groslier. Il localise les fouilles de V. Goloubew en indiquant l'année et parfois le mois des travaux. Ce plan est conservé aux archives de l'EFEO à Paris ( $\left.{ }^{\circ} 2750 \mathrm{CA} / \mathrm{P} / 42\right)$. On doit cependant noter qu'il ne répertorie pas les vestiges uniquement repérés en surface.

23. Une trentaine de mètres pour la chaussée d'Angkor Thom contre plus de cent pour la digue intérieure de CP807 (cf. infra).

24. On peut d'ailleurs douter, vu le fond cartographique disponible en 1932 et la virtualité d'un des deux axes, de la précision de la localisation « exacte » de ce croisement.

25. V. Goloubew a d'ailleurs mis au jour l'année suivante un ouvrage similaire à quelques mètres à l'ouest (1934: 589-590 et fig. 13).

26. Cette digue présente une largeur de $30 \mathrm{~m}$ environ à la base et est distante de $175 \mathrm{~m}$ de la chaussée axiale d'Angkor Thom, dimensions qui divergent complètement de celles des digues de CP807 (cf. infira). 
asséchée, accolée à la face orientale de l'angle du baray avec lequel elle pouvait être liée ${ }^{27}$. On est donc loin de vestiges d'une double levée de terre.

\section{Face ouest}

Dans le schéma proposé par V. Goloubew, la digue orientale du baray occidental recouvre en partie la digue intérieure de Goloupura. Certes, le gabarit actuel de la digue du baray permet une telle hypothèse, même si cela semble plus difficile pour la digue primitive du $\mathrm{XI}^{\mathrm{e}}$ siècle, avant les surélévations qu'elle connut par la suite. Par contre, comme je l'ai indiqué plus haut, l'examen des prises de vues aériennes montre sans ambiguïté qu'aucune digue extérieure n'existait avant le baray: même détruite et soigneusement arasée, une telle digue n'aurait pas manqué de laisser une signature sensible dans le fond du baray, à sec à cet endroit et donc parfaitement visible sur les clichés aériens. Par ailleurs, comme l'a justement noté $\mathrm{Cl}$. Jacques, « il est évident que, si [la] levée extérieure eût existé à l'endroit où la situe V. Goloubew dans le Bàrày occidental, elle eût sans doute servi de digue orientale au Bàrày et non pas la portion équivalente de la levée intérieure " (1978:292).

$\mathrm{V}$. Goloubew relève quelques tronçons de la chaussée axiale ouest du Phnom Bakheng et considère comme « assez vraisemblable » que cette chaussée se soit poursuivie jusqu'au baray, voire même encore plus à l'ouest (1933a : 331). Son plan d'ensemble de Goloupura (1933a : pl. III) montre en effet deux tronçons, l'un commençant au pied du phnom et l'autre s'achevant au droit de la douve d'Angkor Thom. Il indique aussi une amorce de la chaussée à l'angle sud-est du baray ${ }^{28}$ ( $c f$. plan 1). Actuellement, rien ne confirme l'existence d'un tel vestige de chaussée à cet endroit, tant sur le terrain que sur les prises de vues aériennes ou sur les cartes topographiques. Au contraire, ces trois sources s'accordent pour suggérer que la chaussée axiale ouest du Bakheng s'interrompt $400 \mathrm{~m}$ environ avant d'atteindre le baray ${ }^{29}$. 11 semble donc que cette chaussée n'a jamais atteint la digue intérieure de Goloupura.

\section{Face sud}

$\mathrm{Au}$ sud de l'angle sud-est du baray, nous atteignons maintenant les deux digues CP807, les levées de terres de «l'angle sud-ouest» de Goloupura qui servent de " modèle » et auprès desquelles on peut comparer les autres tronçons restitués. Ces digues avaient été prospectées à dos d'éléphant par $\mathrm{H}$. Marchal et V. Goloubew en novembre 1931 " pour rechercher si, comme au Baray Oriental et aux angles de l'enceinte de la ville de Jayavarman VII, des édicules ou bornes inscrites ne s'élevaient pas là, cet angle sudouest étant actuellement, si on adopte l'hypothèse Goloubew, le seul qu'on puisse nettement repérer» $(R C A$ 11/31). Bien que cette zone soit considérée comme étant susceptible d'avoir été la moins altérée, et donc de fournir le plus de témoignages,

27. La digue du baray présente une franche coupure qui semble avoir été remblayée en partie centrale pour la réalisation de la piste qui suit le sommet de la digue.

28. H. Marchal accompagne encore V. Goloubew le 4 octobre 1932 lorsqu'il prospecte cette zone ; il note aussi cette amorce de digue « adhérant à l'angle du baray mais interrompue tout de suite par des rizières » $(J F 10: 11)$.

29. Par ailleurs, les cartes permettent de noter que l'axe du Bakheng ne coïncide pas exactement avec l'alignement de la berge sud du barav, détail qui n'avait pas échappé à H. Marchal ( $c f$. croquis $J F$ $9: 62$ ). 
V. Goloubew et $\mathrm{H}$. Marchal y ont avec peine repéré quelques vagues débris épars ${ }^{30}$. Sur le moment, H. Marchal estime que «tous ces débris, s'ils ne sont pas absolument probants pour la thèse de Goloubew, confirment cependant la possibilité d'un ancien prasat à cet endroit dû à Yaśovarman ». Les sondages qu'il fait réaliser deux mois plus tard ne donnent cependant aucun résultat sinon le repérage à Srè Daun Sâr CP873 d'autres rares blocs épars à l'évidence déplacés, amenant ainsi $\mathrm{H}$. Marchal à douter finalement de l'existence d'une quelconque structure à l'angle de CP807 ${ }^{31}$. Le résultat de mes prospections sur CP807 fut assez similaire : seuls quelques rares briques et tessons peu significatifs peuvent être repérés près de l'angle extérieur CP784. Mais ils n'indiquent ni structure, ni même une réelle occupation des digues puisqu'ils peuvent aussi bien provenir de creusements nécessaires à l'édification de celles-ci ( $c f$. infra). Enfin, les quelques débris retrouvés en 1931 peuvent même suggérer une date postérieure pour les digues CP807 ${ }^{32}$.

Les cartographies topographiques détaillées permettent aussi d'émettre plusieurs remarques sur la configuration de CP807. Il apparaît que les deux digues CP807 ne présentent pas exactement la même section l'une par rapport à l'autre ${ }^{33}$, et que la digue intérieure est moins large sur son tronçon est-ouest que sur son retour nord-sud vers le baray occidental (Garami et Kertal, 1993: 36 et fig. 34-35). Par ailleurs, la distance qui sépare les digues est légèrement mais sensiblement différente : $310 / 300 \mathrm{~m}$ sur la face occidentale et 290/280 $\mathrm{m}$ sur celle du sud (distance entre sommets), différence qui semble assez surprenante si ces deux digues et la «douve» qu'elles bordent avaient formé une enceinte.

Les prospections m'ont aussi amené à repérer un site inédit à l'angle sud-ouest de CP807, le Kôk Daun Bos CP654 qui, s'il ne montre aucun vestige de structure, possède

30. "[À l'] angle intérieur (...) de menus débris de briques et au bas de la digue quelques blocs minuscules de grès sans forme nette », « à l'angle extérieur un [fragment de] colonnette ronde du genre de celle de Sambor (...), débris de briques et quelques fragments de latérite (...) des blocs sculptés ou taillés en grès mais trop enterrés pour qu'on puisse préciser leur identité (...) un morceau sculpté encastré dans les racines d'un arbre (pierre d'antéfixe ou motif d'entrepilastre de l'art d'Indravarman) », "plus au nord (...) une petite statue de femme debout » (JF $9: 56-57)$.

31. Seuls sont mis au jour au nord de l'angle de la « levée de terre extérieure [...] un petit sommet de fronton triangulaire à motif ornemental classique, [et] une antéfixe plate décorée d'un dvarapala dans la pose de ceux de Lolei ». Marchal note encore « en revenant [sur la digue intérieure] l'existence de 2 Nak Ta [à Srè Daun Sâr CP873] (...). Ces pierres (...) proviennent probablement de quelque prasat classique ». Et de s'interroger: «Peut-être en est-il de même des pierres sculptées près de l'angle de la digue ?» (JF $9: 86-87)$.

32. Quelques vestiges mis au jour («l'antéfixe plate ») peuvent certes provenir en partie de l'époque du Bakheng. Mais, H. Marchal le note lui-même, ces vestiges demeurent particulièrement ténus pour suggérer l'existence d'un sanctuaire de Yaśovarman et dater les digues, d'autant qu'aucune structure n'a pu être dégagéc. Quant à la colonnette qui «serait du genre de celle de Sambor », le croquis qui en est donné (.JF $9: 57$ ) pourrait aussi bien montrer une balustre d'une baie haute (bibliothèque, ou salle longue à partir de la fin du $x^{*}$ siècle ?). Par contre, d'autres éléments (la «pierre d'antéfixe ou motif d'entrepilastre de l'art d'Indravarman», le «sommet de fronton triangulaire », une pierre longue moulurée en grès qui semble avoir reçu des épis de faîtage car le dessus est entaillé en mortaise) pourraient indiquer non seulement une époque plus tardive, mais aussi que les structures dont proviennent ces éléments ont justement pu être détruites lors de la réalisation de ces levées de terre. Ces dernières seraient donc au moins de l'époque classique, « post-Indravarman ».

33. Il a bien sûr ici été tenu compte du fait qu'une partie orientale de la «digue extérieure » a été profondément altérée lors de la réalisation de l'aéroport voisin dans les années 1945-1946 (cf. plan 5 et fig. 1 , coupe 2 ). 
une configuration toute particulière ${ }^{34}$ qui ne se retrouve dans la région d'Angkor qu'à CP653 présentant des vestiges d'un monastère tardif. La similitude entre ces deux sites concerne d'ailleurs aussi leur localisation : CP653 est situé à l'ouest de l'angle sud-ouest de la douve d'Angkor Vat, de même que CP654 par rapport à CP807. Interpréter cette similitude est toutefois nettement prématuré sans investigations approfondies sur ces deux sites; aussi se bornera-t-on ici à souligner le lien éventuel que peuvent entretenir Angkor Vat et CP807 via l'existence d'une configuration spéciale à leur angle sud-ouest.

Avant d'aborder la face orientale de Goloupura, mentionnons encore que la chaussée sud du Bakheng, dont des vestiges ont été rencontrés jusqu'à la hauteur de l'axe occidental d'Angkor Vat ${ }^{35}$, ne semble pas se poursuivre beaucoup plus au sud. V. Goloubew semble justifier cette disparition en estimant « que la construction de la route conduisant à la porte sud d'Angkor Thom avait entraîné de bonne heure, sans doute dès la fin du XII ${ }^{c}$ siècle, l'abandon de la chaussée établie sous Yaśovarman » (1933a : 332). Il est pourtant douteux qu'un tel abandon puisse être la cause de la disparition de toute trace de cette chaussée, étant exclu que celle-ci ait pu servir à celle d'Angkor Thom, distante de plus de $350 \mathrm{~m}$. Ici encore, les prises de vues aériennes confirment les renseignements cartographiques et les observations de terrain, et indiquent que la chaussée sud du Bakheng n'atteint pas la face sud de Goloupura.

\section{Face est}

La face orientale, la dernière face de Goloupura, est celle où le moins de vestiges ont été repérés par V. Goloubew; au point que suivant l'avis de H. Parmentier, il envisage que la digue extérieure «n'ait pas été prévue par Yaśovarman et ses conseillers techniques, la protection de la capitale étant assurée, du côté est, par le cours détourné de la rivière de Siemreap » (1933a : 340). Outre que l'on a certaines raisons de penser que cette rivière a été canalisée - ou même créée - à une époque nettement plus tardive que le $\mathrm{X}^{\mathrm{c}}$ siècle (Pottier, 1999: 100-101 et 168), son utilité défensive est contestable : les «conseillers techniques » de Yaśovarman ne pouvaient se contenter de cette maigre coupure large d'à peine $30 \mathrm{~m}$, sans levée de terre particulière et aisément franchissable. Par ailleurs, imaginer une douve autour de la digue intérieure de Goloupura se heurte à la présence d'au moins deux sanctuaires installés sur son implantation théorique : un site sans nom CP870 à l'extrémité de la face sud de CP807, près de l'angle sud-est de Goloupura ${ }^{36}$, et bien sûr Kapilapura 714 au nord-est

34. Ce site CP654 présente actuellement neuf terre-pleins rectangulaires ( $50 \times 40-35 \mathrm{~m}$ ) couverts de broussailles qui dominent d'un mètre les rizières environnantes. Les terre-pleins sont alignés en trois « lignes » est-ouest espacées d'environ $20 \mathrm{~m}$, et trois «colonnes » nord-sud espacées d'environ $30 \mathrm{~m}$. L'existence d'une quatrième «ligne » au nord est suggérée par les prises de vues aériennes. Bien que peu sensibles au sol car présentant un front continu de broussailles «impénétrables», ces terre-pleins septentrionaux semblent bien faire partie d'une configuration d'origine puisque les photographies aériennes montrent la trace presque carrée $(270 \times 290 \mathrm{~m})$ d'une dépression (douve ?, en rizière actuellement) qui ceint l'ensemble des douze terre-pleins (quatre lignes et trois colonnes).

35. En 1931, lors des débroussaillements préliminaires à la réalisation d'une piste dans l'axe majeur d'Angkor Vat menant à l'aéroport, H. Marchal note « la découverte, à environ 300 mètres des douves [d'Angkor Vat], d'une levée de terre perpendiculaire au temple et qui a dû correspondre à une ancienne chaussée khmère. Il serait désirable de suivre cette chaussée, qu'on ne voit portée sur aucune carte, pour savoir où elle aboutit » ( $R C A$ 08/31). Les prospections qu'il mène l'année suivante avec V. Goloubew $\mathrm{n}$ 'apportent aucun élément nouveau.

36. Il s'agit des vestiges d'un sanctuaire de brique, avec cadre et piédestal en grès et fondation en latérite, établi sur un tertre assez élevé. Il présente des colonnettes épannelées octogonales, mais aucun 
d'Angkor Vat ${ }^{37}$. En fait, il est clair qu'aucun élément ne permet d'envisager une digue extérieure, ni même une douve sur cette face. Le schéma de V. Goloubew s'appuie donc ici principalement sur une seule digue intérieure, identifiée au bourrelet qui longe la face orientale des douves d'Angkor Vat ${ }^{38}$ puis se prolonge un peu au nord. Les prospections sont encore difficiles dans cette zone couverte de broussailles et tant les prises de vues aériennes que les cartes topographiques y sont peu précises. Cependant, le relevé topographique réalisé en 1934 a montré que cette levée de terre tend à s'estomper vers le nord et ne se poursuit pas au-delà de l'axe oriental du Bakheng; à partir de ce niveau, seule une seconde digue remonte vers le nord jusqu'à la douve méridionale d'Angkor Thom, mais elle est décalée de " 20 à 30 mètres » vers l'ouest (Goloubew 1934: 599). Plus au nord, V. Goloubew "hésite à voir dans la "levée garnie E. d'Angkor Thom" le prolongement vers le nord [de la digue intérieure, car celle-ci] paraît plutôt avoir affecté un tracé correspondant au bord extérieur de la douve qui est à l'est d'Angkor Thom » (1933a: 340). Il n'y a donc pas de vestige réellement probant sur la face orientale de Goloupura, et la seule levée de terre de quelque ampleur, celle aux abords d'Angkor Vat, est étroitement associée à ce temple, et lui est donc très probablement contemporaine.

Enfin, sur cette face orientale, l'existence d'une longue chaussée dans l'axe du Bakheng apparaît comme plus assurée. V. Goloubew l'a amplement explorée et restituée jusqu'à la rivière de Siemreap en s'appuyant notamment sur la découverte près de celle-ci des «vestiges d'un prasat » GT82 hâtivement interprété comme un sanctuaire destiné au culte de la Gañgā (1933a: 326-330). Les levés topographiques réalisés en 1934 montrèrent cependant que ce site n'était pas situé sur l'axe du Phnom Bakheng mais $150 \mathrm{~m}$ au nord (1934: 599 ; $R C A$ 02/34). D'ailleurs rien de ce qui y a été mis au jour n'implique que la rivière ait existé à l'époque de ces vestiges qui, s'ils sont proches de cette dernière, ne lui sont pas particulièrement liés. G. Trouvé a prospecté à la même époque la région située à l'est de la rivière et il ressort de son travail que la chaussée axiale a pu se prolonger vers l'est et rejoindre la chaussée CP793 menant à Beng Méaléa (1933 : 1124-1126) ${ }^{39}$. Finalement, il apparaît que la chaussée axiale du Bakheng a pu exister et se prolonger à l'est, mais elle ne montre aucun aménagement particulier à l'intersection tant de la rivière que du tracé de Goloupura.

Au terme de cet examen détaillé des vestiges de l'enceinte de Goloupura, force est de rejoindre B. P. Groslier lorsqu'il indique abruptement que le «tracé supposé au N. E., sur tout le parcours nord et est, n'existe pas et rien n'apporte la moindre confirmation » (1979: 182).

\footnotetext{
élément ne m'a semblé pouvoir permettre d'avancer une datation précise. Que ce sanctuaire soit contemporain de Yaśovarman ou postérieur, son emplacement tend à contredire l'existence de l'angle sud-est de Goloupura. En effet, si l'incompatibilité est évidente s'il date du début du x $x^{c}$ siècle, il est aussi diffícile de considérer qu'un sanctuaire ait été établi, plus tardivement, "à cheval » sur la douve et la digue extérieure de Goloupura, que cela soit avant qu'elles aient été arasées ou après.

37. Kapilapura a été " découvert » et dégagé en 1924 par H. Marchal et date vraisemblablement du $\mathrm{X}^{*}$ siècle. C'est d'ailleurs V. Goloubew qui avait été chargé en juin 1924 d'y réaliser l'estampage de K. 579 qui porte la date de 968 A.D. (RCA 06/24).

38. Cette levée de terre, assez irrégulière, est large d'environ $100 \mathrm{~m}$. Sur la face sud d'Angkor Vat, elle semble nettement plus étroite, seulement $50 \mathrm{~m}$. Les prises de vues aériennes et les plans topographiques montrent par ailleurs que le bourrelet de la face orientale d'Angkor Vat diffère nettement et n'est pas aligné avec la digue qui se poursuit vers le sud et rejoint l'extrémité orientale de CP807.

39. Bien qu'en 1933, aucune continuité de la chaussée sur la rive gauche n'est indiquée (Goloubew, 1933a : pl. III), elle est suggérée sur la carte de G. Trouvé de 1934 (Dumarçay \& Pottier, 1993 : pl. 1).
} 


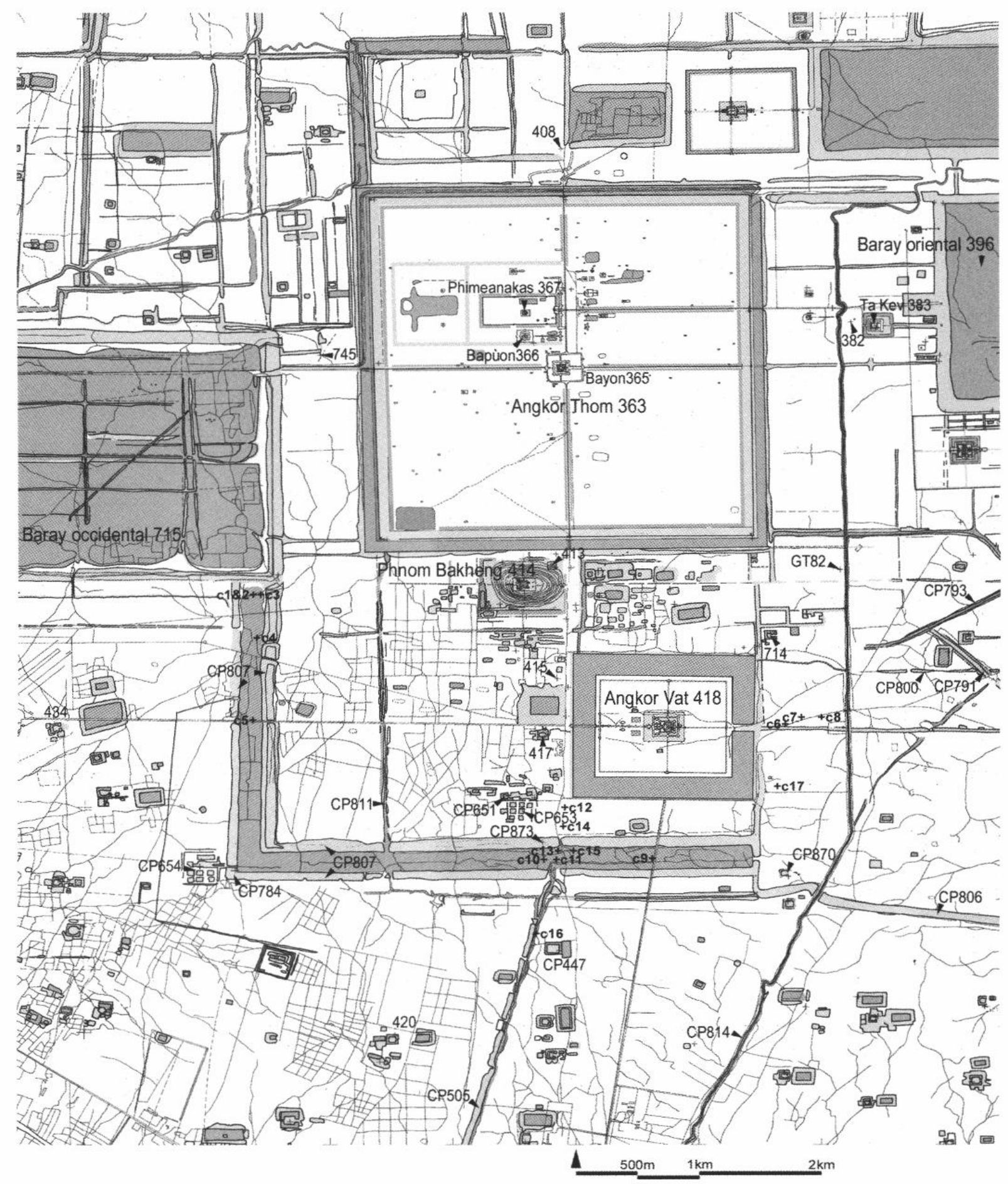

Plan 3 - Environs du Phnom Bakheng : localisation des sites archéologiques et des carottages. (EFEO/Univ. Sydney 1999-2000) 


\section{Éléments stratigraphiques}

La région environnante du Phnom Bakheng a longuement été occupée et il est probable que de nombreuses structures du $\mathrm{X}^{\mathrm{c}}$ siècle ont été profondément altérées ou recouvertes lors des occupations suivantes, en particulier lors de la réalisation d'Angkor Thom. Si l'on peut avancer cette explication pour justifier la disparition au sol de quelques tronçons de Goloupura, elle n'est guère satisfaisante face à une telle pauvreté de vestiges, à moins que des profils stratigraphiques témoignent sans équivoque d'états antérieurs. Malheureusement, nous n'avons pas retrouvé d'informations de ce type recueillies dans les années 1930. Tout juste peut-on rappeler que $\mathrm{H}$. Marchal avait remarqué à propos de vestiges dégagés par V. Goloubew au sud de la chaussée occidentale du Bayon, que ces structures, en plus d'être « vagues », étaient toutes " presque à fleur de sol, à peine 0,50 ou $0,60[\mathrm{~m}]$ de terres à enlever » $(J F 10: 31)$. Cette remarque constituait à son avis une objection contre le rattachement de ces vestiges au $\mathrm{X}^{\mathrm{e}}$ siècle. Cependant, l'argument de $\mathrm{H}$. Marchal ne peut être entièrement retenu puisqu'il comparait cette faible profondeur à celle de l'enceinte du palais royal, dont on sait depuis que l'épaisseur des couches n'est guère représentative du reste d'Angkor Thom.

Il reste que l'absence en surface de traces de Goloupura pourrait être contournée par des informations stratigraphiques susceptibles de conserver au moins la signature d'une douve. B. P. Groslier affirme à ce propos que «les très nombreux sondages stratigraphiques pratiqués du Bayon au Roi Lépreux, entre 1960 et 1972, n’ont jamais révélé une "douve nord" de Yaśodharāpura, qui n'aurait pas manqué d'apparaître » (1979:182). Vu l'ampleur des travaux dirigés par B. P. Groslier dans cette zone, on considérera le poids d'un tel argument même si ces coupes stratigraphiques sont restées inédites, hormis celles des sondages réalisés par J. Dumarçay au Bayon (Dumarçay et Groslier, 1973 : 1721 , pl. XI-XII). Ces derniers sondages ne montrent en l'occurrence aucune trace susceptible d'étayer l'hypothèse de V. Goloubew ${ }^{40}$. Récemment, des campagnes de prospections par carottages ont été réalisées par R. Exaltus dans le cadre du programme de recherches archéologiques sur Angkor Thom, intitulé «Études urbaines » et dirigé par J. Gaucher. Ces carottages ont mis en évidence la présence d'un ancien plan d'eau au sud du Bapùon, mais il ne se poursuit pas à l'est du Bayon et son étendue ne correspond guère à l'implantation de l'éventuelle douve nord de Goloupura (Gaucher, 1996: 12 et pl. 5 ; 1997 : pl. 5). Les indications stratigraphiques disponibles tendent donc à contredire l'existence de la partie nord de Goloupura.

Aucune donnée stratigraphique ne semblait exister sur les parties restantes de la douve de Goloupura. Aussi, lors d'une campagne de carottages pilotes réalisée dans le cadre de la phase préliminaire d'une étude conjointe sur le développement du territoire angkorien, $\mathrm{R}$. Fletcher et moi-même avons réalisé en décembre 1999 quelques carottages à certains emplacements de la douve supposée de Goloupura ${ }^{41}$. Le nombre réduit de carottages n'a

40. L'équipe de JSA (Japanese Government team for Safeguarding Angkor) a réalisé au début de l'année 2000 un sondage nord-sud au Bayon, depuis la première enceinte jusqu'à la route actuelle autour du temple. Longue d'environ $80 \mathrm{~m}$, cette remarquable section a été implantée entre les sondages 3 et 8 de J. Dumarçay et montre une stratigraphie globalement assez similaire à ces derniers. Aucune trace d'une éventuelle douve n'est visible.

41. Cette série de carottages pilotes a été financée par l'EFEO et l'Australian Research Council. Les détails de ces carottages dépassent le cadre du présent article et feront l'objet d'un rapport spécifique. Nous remercions le projet «Études urbaines » pour avoir aimablement mis une tarière à la disposition de cette phase préliminaire. 
permis d'obtenir qu'une vision très succincte et donc une compréhension partielle des profils stratigraphiques des zones sondées. Par ailleurs, la rapidité de l'évolution des sols en milieu tropical pouvant entraîner la disparition des couches d'occupations non architecturales, une certaine prudence s'impose quant à l'interprétation des niveaux repérés ${ }^{42}$. Il reste que, parmi les carottages réalisés entre les digues de CP807 ( $c f$. localisation sur plan 3), plusieurs ont été réalisés au sud de la digue du baray occidental afin d'y chercher les traces éventuelles des deux phases de sédimentation qui auraient pu témoigner de la postériorité de cette digue dans l'hypothèse de V. Goloubew ( $\mathrm{cl}$ à $\mathrm{c} 4)$ : ils n'y ont rien montré de semblable. Par ailleurs, aucune trace de douve n'est perceptible dans les carottages de la face orientale (c6 à $\mathrm{c} 8$ et $\mathrm{c} 17)$. Ceux réalisés sur les faces ouest et sud de la «douve » de Goloupura n'ont montré qu'un niveau de dépôt (c2 à c5, c9, c10 et c15), hormis deux carottages situés dans le prolongement d'un canal CP505 dont la signature retrouvée sous le fond de la « douve » indique qu'il est antérieur à cette dernière (c1l et c13, cf. infra). Les résultats de ces carottages ne montrent finalement aucune trace caractéristique permettant de soutenir l'hypothèse de V. Goloubew; au contraire, ils fournissent les éléments pour situer la réalisation de l'ouvrage CP807 à unc époque nettement postérieure ( $c f$. infra).

\section{Parcellaires anciens et données épigraphiques}

Des éléments d'un autre ordre apportent encore des arguments qui contredisent l'existence de Goloupura, et plus particulièrement celle de CP807 à l'époque de Yaśovarman. L'analyse des divers clichés aériens pris depuis les années 1930 montre la présence d'un parcellaire géométrisé induit par le baray oriental et situé au sud de celui$\mathrm{ci}^{43}$; je considère d'ailleurs que, dans sa majeure partie, ce parcellement est contemporain du baray (Pottier, 1999: 117-119). Cette masse parcellaire se poursuit à l'ouest jusqu'à englober le Phnom Bakheng et s'étendre encore sur $1500 \mathrm{~m}$ vers l'ouest et autant au sud (cf. grisé clair sur plan 4). Ce parcellaire isocline et contemporain ${ }^{44} \mathrm{du}$ Bakheng ne rejoint à aucun moment la double levée de terre CP807, ni donc les faces occidentale et méridionale de Goloupura. Dans les parties restantes de l'enceinte supposée, le parcellaire ne tient qu'exceptionnellement compte de la digue intérieure, ce qui ne manquerait pas de surprendre si CP807 avait été l'enceinte d'une ville. En fait, les traces parcellaires semblent essentiellement indépendantes de CP807 : en deux endroits au moins à l'ouest, ces parcelles apparaissent dans la continuité de parcellaires situés à l'extérieur de l'« enceinte » (cf. a \& b sur plan 4), indiquant que ces masses parcellaires sont antérieures à Goloupura.

Nous avons ici l'opportunité de trouver dans l'épigraphie confirmation de la télédétection. Aux Prasat Trapéang Ropou 434 et Prasat Kôk Châk 420, deux temples voisins du phnom et situés à l'extérieur de CP807, les inscriptions rapportent des limites de terrain mitoyennes à ceux du vnam kantāl (le Phnom Bakheng). Cette coïncidence est assez heureuse pour être notée et pour que l'on revienne ici sur le détail de ces deux inscriptions afin d'y chercher une éventuelle allusion aux deux digues de CP807, élément

42. Marc Franiatte a bien voulu nous faire partager son avis sur ce sujet ; qu'il en soit ici vivement remercié.

43. J'emploie ici le mot «parcellaire » non pas dans un sens cadastral ou foncier, mais dans un sens morphologique, en tant que dessin des limites agraires constatées au sol, correspondant en majorité à la trame de rizières actives et fossiles.

44. On note en particulier l'insertion de toutes les structures au pied du phnom dans ce parcellement. 
topographique notable qui, s'il avait existé à l'époque de ces inscriptions, n'aurait pas manqué d'apparaître dans la délimitation entre les terrains du vnam kantāl et ses voisins.

\section{Prasat Trapéang Ropou 434}

Ce temple, dont les vestiges indiquent qu'il fut érigé vers la fin du $\mathrm{X}^{\mathrm{e}}$ siècle, présente deux inscriptions qui offrent des repères datés: K. 691 sur le piédroit sud de l'édifice annexe (bibliothèque), qui relate «l'installation du Feu sacré » (vraisemblablement dans cet édifice) en 1002 (Cœdès, 1952 : 151) ; et K 690 gravée sur les piédroits de la tour sud. Dans cette seconde inscription, Cœdès « reconnaît la fin d'une énumération de terres dont il n'y a guère à retenir que le nom du Vāp Śikhavindu, auteur d'une inscription de 921 à Koh Ker » (1964b: 91). L'inscription se compose de deux parties distinctes : la première concernait "semble-t-il (...) un dispositif de fondation» dans lequel est mentionné Candrapura déjà indiqué dans K. 691 ; la seconde «est une ordonnance royale, peut-être de Rājendravarman, chargeant deux dignitaires de faire mesurer la contenance des terres, ou d'une terre, de Vnam Kantāl [Phnom Bakheng], dans le but probable d'en attribuer tout ou partie au dieu de Travān Rvau ", l'actuel Trapéang Ropou dont le nom s'est remarquablement perpétué. Le texte précise que deux personnes sont chargées «de venir voir les dimensions exactes de la terre de Vnam Kantāl ». Suit alors la mention incomplète d'une restitution «à nouveau au dieu à Travān Rvau ». Malgré les lacunes, il est clair qu'un litige de limite de terrain opposait le sanctuaire de Trapéang Ropou à celui du Phnom Bakheng. À la différence de nombreuses inscriptions qui évoquent de tels litiges sur des terres parfois fort éloignées du sanctuaire auquel elles sont rattachées, la proximité des sanctuaires suggère ici que les terres en question étaient mitoyennes ( $f$. plan 4). Si l'enceinte CP807 existait à l'époque, on ne peut qu'être surpris par le fait qu'elle ne soit pas mentionnée ici dans cette délimitation qui semble d'ailleurs assez confuse pour motiver la venue d'inspecteurs. Le parcellaire est encore assez clair dans cette zone ; celui du Prasat Trapéang Ropou, nettement désaxé vers le nord-est $\left(78^{\circ}\right)$, se poursuit à l'intérieur de CP807 (a sur plan 4) jusqu'à rejoindre effectivement le parcellaire orienté autour du Phnom Bakheng: ces deux masses parcellaires étaient donc mitoyennes et CP807 apparaît comme un rajout ultérieur sur le parcellaire de Trapéang Ropou.

\section{Prasat Kôk Châk 420}

Les vestiges architecturaux - les linteaux en particulier - suggèrent que les deux tours du Prasat Kôk Châk ont été édifiées vers la fin du $\mathrm{X}^{\mathrm{e}}$ siècle, alors que le temple était une fondation royale. La majeure partie de la statuaire retrouvée lors d'un dégagement en 1951 semble d'ailleurs dater de cette époque et aucune modification n'a laissé de trace postérieure. Les inscriptions de ce temple, K. 521, ont été traduites par Cœdès (1952: 167170). Elles consistent en deux « textes indépendants [mais] écrits de la même main et en rapport étroit l'un avec l'autre. Ils doivent être sensiblement postérieurs à Süryavarman $I^{\mathrm{Ir}} \gg(1002-1050)$ et à l'érection des sanctuaires. L'inscription de la tour sud relate l'origine de la fondation de ce sanctuaire de Śakabrāhmana suite à un rêve divinatoire de Jayavarman III, qui avait perdu un éléphant dans la forêt recouvrant la région à cette époque. Après débroussaillement, fut alors consacré le Viṣnugrāma, nom ancien du site de Prasat Kôk Châk. On passera brièvement sur le détail du changement de statut de ce site et de son terrain environnant en mentionnant juste qu'il était une fondation et un domaine royal jusque sous le règne de Sūryavarman $I^{\mathrm{er}}$ durant lequel il est " offert à la reine Vỉralakṣmị [à laquelle] un ancêtre de l'auteur de l'inscription rappela qu'il restait à y planter les bornes. (...) Le texte donne la composition de la commission d'abornement et indique les limites des terrains abornés ». 
Transcrites, mais non traduites par Cœdès, les limites indiquées à la fin de l'inscription mentionnent cependant que l'un des terrains va, au nord, jusqu'aux terres du Phnom Bakheng (1. 12 : vnam kantāl) ${ }^{45}$. Encore plus clairement ici qu'au Prasat Trapéang Ropou voisin, l'inscription montre que le terroir de Viṣnugrāma était mitoyen de celui du vnam kantāl / Phnom Bakheng ${ }^{46}$. Et ici encore, nulle mention n'est faite des limites de la ville, ou des digues CP807 : au contraire, la délimitation de la mitoyenneté nécessite un comité de 13 personnes pour aller planter des bornes de pierre et de bois. L'épigraphie du Prasat Kôk Châk suggère donc elle aussi que CP807 n'existait pas avant la seconde moitié du XI ${ }^{\mathrm{e}}$ siècle et ne saurait donc constituer un vestige d'une Goloupura du $\mathrm{X}^{\mathrm{e}}$ siècle.

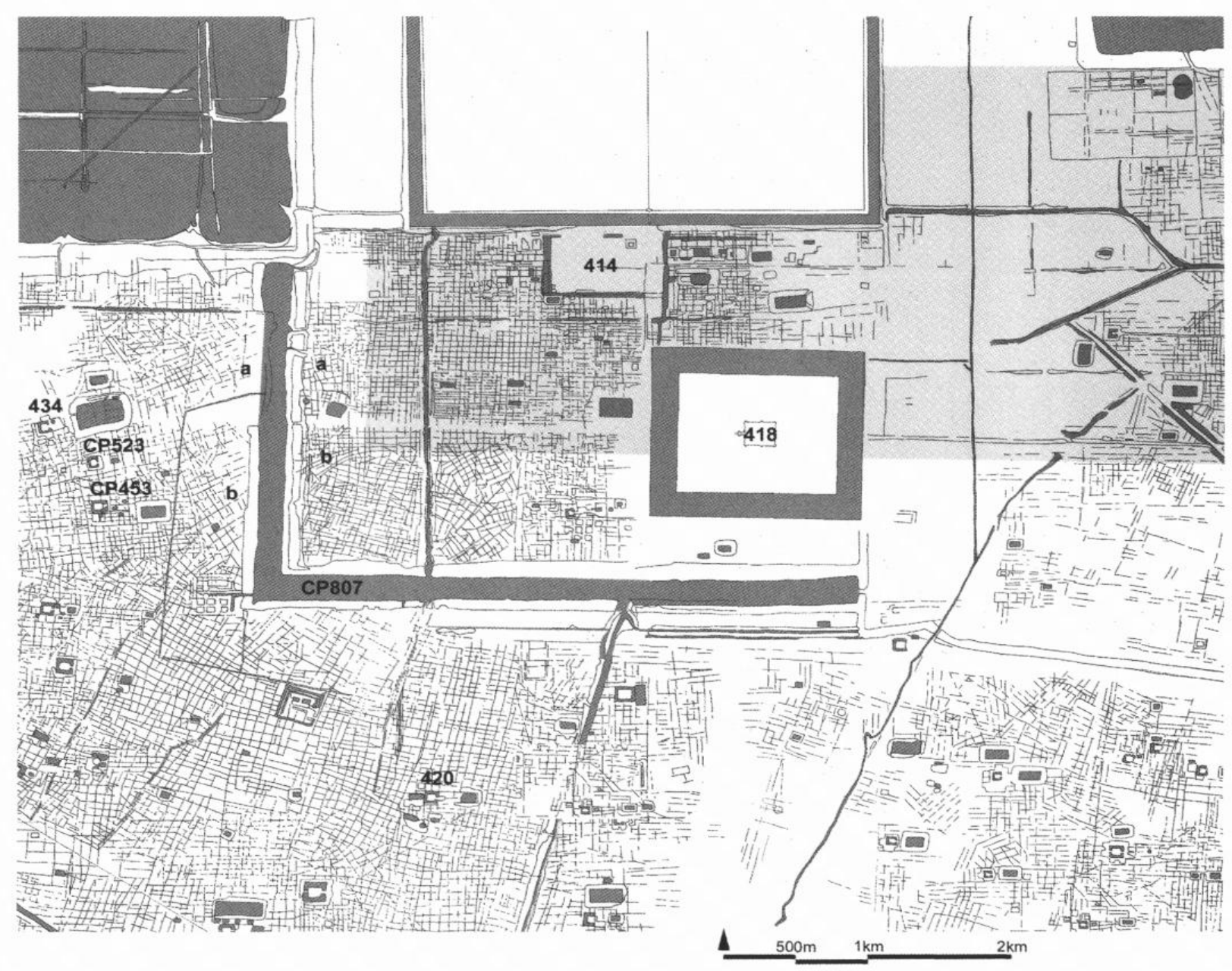

Plan 4 - Parcellaire des environs du Phnom Bakheng.

45. Cette lecture nous a été aimablement confirmée par Claude Jacques.

46. Si le texte de l'inscription semble plus clair, l'analyse du parcellaire ancien l'est moins; la limite sud de celui isocline au Bakheng est nette au sud de l'actuelle chaussée ouest d'Angkor Vat, mais on n'observe pas de trace évidente de la continuité du parcellaire du Prasat Kôk Châk au nord de CP807. 


\section{Une fonction pour l'angle sud-ouest de Goloupura?}

La logique d'assemblage, l'observation des vestiges, les données stratigraphiques et le recoupement du tissu parcellaire ancien avec les données épigraphiques s'opposent à la matérialisation de l'enceinte de Goloupura. Cependant, les digues de CP807 existent bel et bien et cet ouvrage reste légèrement énigmatique, tant dans sa fonction que dans sa datation. Nous avons vu plus haut qu'un quelconque aspect défensif ne peut guère être retenu même si l'on considère l'ampleur de cet ouvrage. D'ailleurs, la région d'Angkor ne connaît aucun ouvrage comparable dont la fonction défensive puisse être attestée, et l'on ne peut que rester circonspect devant le réel potentiel stratégique d'un seul angle sudouest. Sensible à l'aspect hydraulique des infrastructures, B. P. Groslier a considéré que CP807 constituait une «"vallée artificielle" : circuit forcé permettant à la fois de distribuer l'eau captée, de cultiver les rizières et de pratiquer les semis ou encore des cultures dérobées » (1979: 183). Il rappelle que CP807 «n'a pu fonctionner que le baray occidental existant. Dès lors sa fonction devient claire : par le jeu alterné du trop plein de ce baray et des crues du St Siemreap (captées par la digue-barrage au sud-est), il peut être rempli quand de besoin, assurer les semis, irriguer les rizières qu'il renferme ". Si l'on peut partager les observations de cet auteur pour ce qui est de l'alimentation de CP807, une telle fonction semble toutefois douteuse pour un ouvrage d'une telle ampleur. Il est difficile en effet d'imaginer que ces digues atteignant parfois $6 \mathrm{~m}$ de hauteur n'ont été conçues que pour assurer une nappe d'eau pour des premiers semis, aussi cruciaux soientils. De plus, cette fonction ne justifie pas le plan en « $L$ » très particulier de CP807. Enfin, elle ignore une caractéristique essentielle de cette "vallée artificielle »: les documents topographiques indiquent que l'espace entre les deux digues de CP807 est nettement en contrebas des terrains environnants, de 1 à $2 \mathrm{~m}$ actuellement ( $c f$. plan 5 et fig. 1$)^{47}$ et sensiblement plus à l'époque angkorienne. Ceci suggère que les deux digues furent réalisées avec les terres du creusement de la douve, et que cette douve devait être en eau toute l'année, comme celle d'Angkor Vat par exemple, dont le niveau est asseż similaire. Il n'est donc pas envisageable que cet espace ait pu servir aux premiers semis de riz, ni même à la culture du riz.

47. Les cotes portées sur les plans topographiques de 1962 (Dumarçay \& Pottier, 1993 : pl. 9) sont peu nombreuses à l'intérieur de la « douve » de CP807 et ne permettent guère de mettre en évidence cette caractéristique. En particulier, la cote de 18,3 m indiquée au centre de l'angle sud-ouest de la «douve » ne nous semble aucunement représentative du niveau du fond de la douve à cet endroit; il s'agit au mieux de celui d'une petite éminence où poussent actuellement quelques palmiers à sucre et qui dut être utilisée comme point de repère lors de l'élaboration des données altimétriques. Ce point est d'ailleurs désigné sur le calque de nivellement original ( $n^{\circ} 2690$, archives EFEO Paris) sous le code «cr » signifiant «cote restituée" donc non déterminées sur le terrain ( $\mathrm{F}$. Boldo, ingénieur de l'IGN, communication personnelle). La nouvelle couverture topographique réalisée par JICA est, pour cette zone, plus précise (JICA, 1998 : pl. 5 et 9 au 1/5000 et pl. 6 au 1/10 000). Nous avons complété ces niveaux par quelques points relevés in situ; ceux-ci sont indiqués en italique sur le plan 5. 


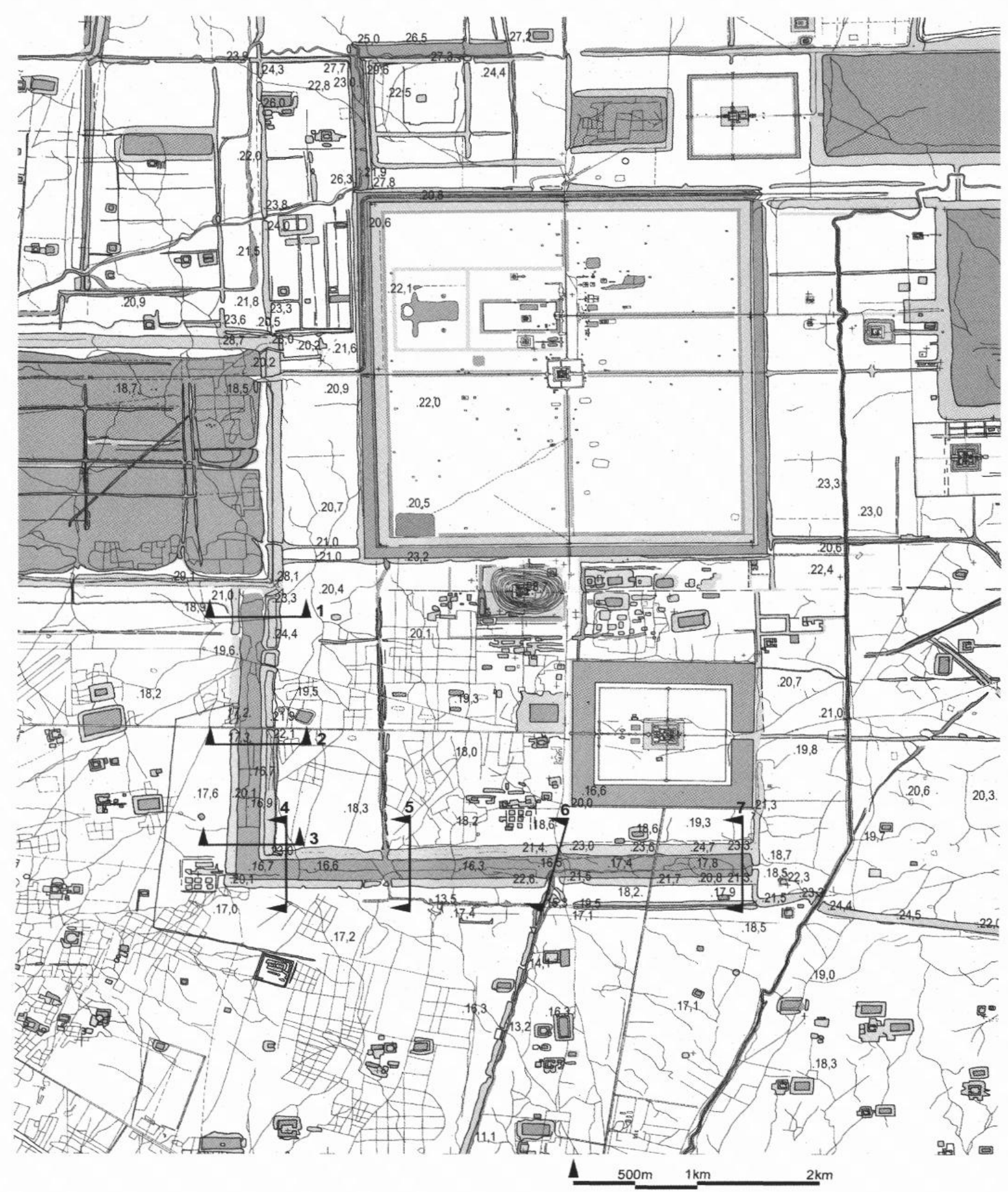

Plan 5 - Environs du Phnom Bakheng : nivellements. 


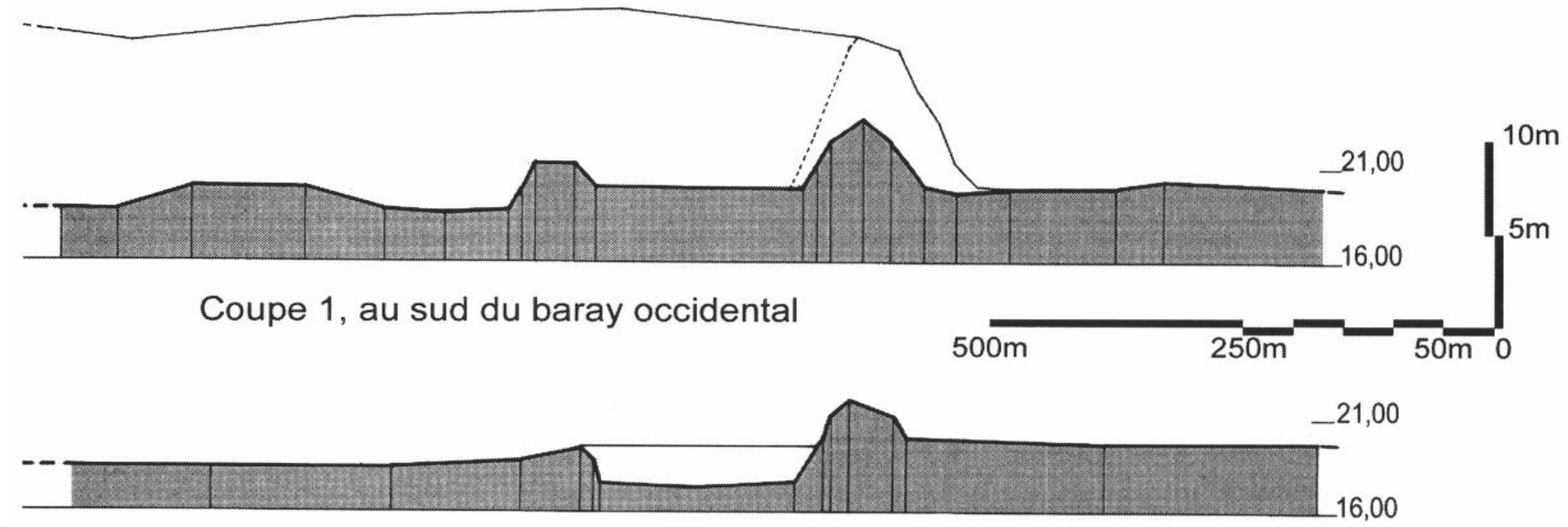

Coupe 2, au sud de la route dans l'axe ouest d'Angkor Vat

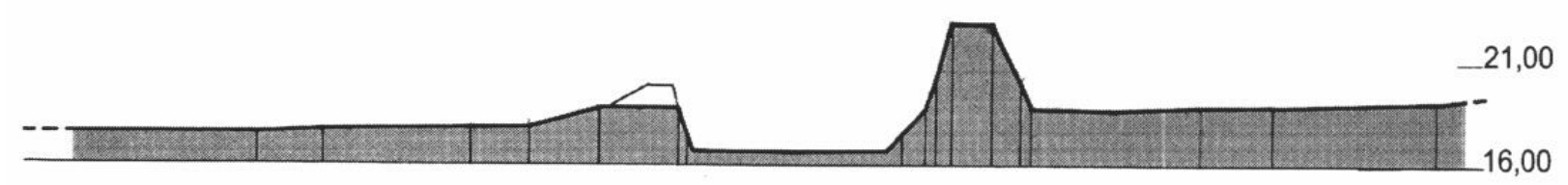

Coupe 3, au nord de l'angle sud ouest

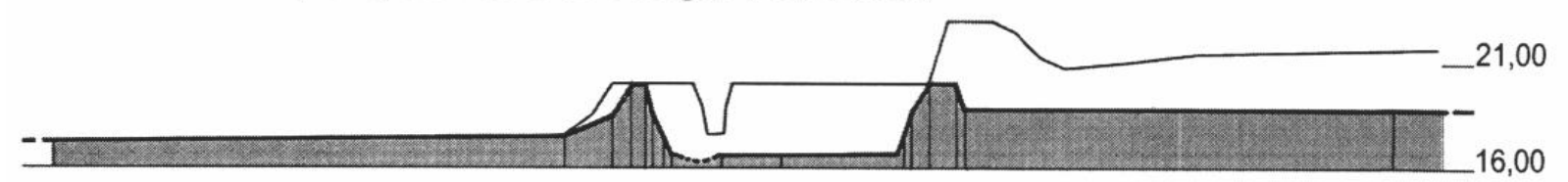

Coupe 4, à l'est de l'angle sud ouest

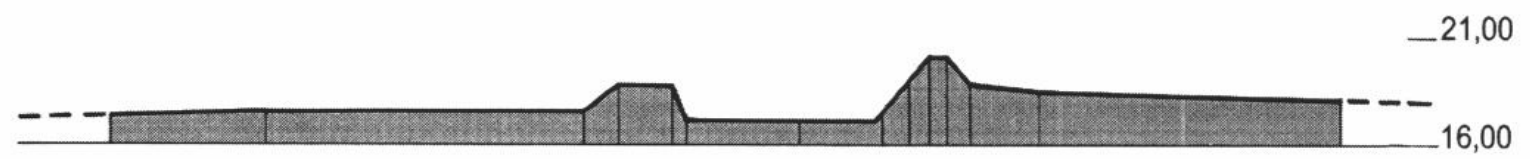

Coupe 5, à l'est du canal CP811

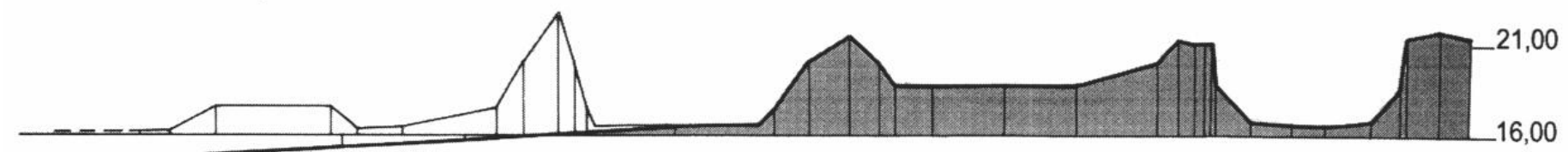

Coupe 6 dans le canal CP505 jusqu'à la douve d'Angkor Vat

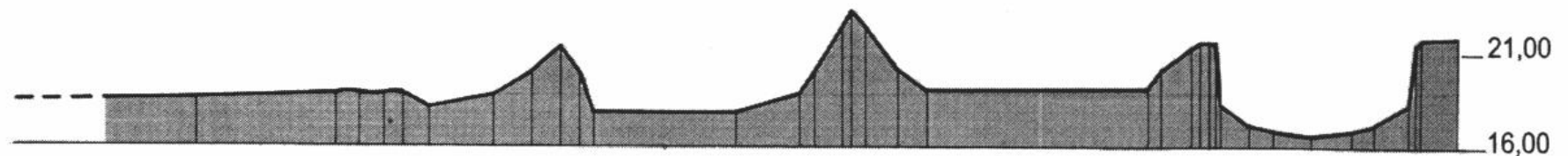

Coupe 7, près de l'extrémité orientale, jusqu'à la douve d'Angkor Vat

Fig. 1 - Sections sur les digues CP807. 
Le cours actuel de la rivière de Siemreap - le stung Siemreap - semble en fait avoir troublé la vision du fonctionnement de la zone étudiée. B. P. Groslier assure certes avoir retrouvé les traces d'un ancien lit de rivière du VII ${ }^{\mathrm{e}}$-VIII ${ }^{\mathrm{e}}$ siècle à l'est de Thommanon 380 (1979 : 165) et d'un second "de l'âge de bronze » aux environs de Baksei Chamkrong 413 ( $R C A$ 1966), alors qu'un troisième a encore été repéré dans les environs du Bayon (Gaucher, 1996: pl. 3-8). Ces indications en partie contradictoires suggèrent que pour l'instant, l'existence, la localisation et le régime d'un stung Siemreap primitif ne sont guère assurés. Remarquons d'ailleurs l'embarras des restitutions d'un cours primitif dans les cartes de B. P. Groslier (1979 : carte 2$)^{48}$. Finalement, les prises de vues aériennes ne montrent des traces "naturelles » en méandre qu'en aval, à partir des environs de Vat Athvéar 422 : encore sont-elles situées au débouché de tronçons artificiels... Aussi, si l'on doit considérer que quelques cours d'eau ont pu exister avant l'installation de Yaśovarman aux environs du Phnom Bakheng, on peut douter de la présence d'une véritable rivière permanente telle que le stung Siemreap dont les seules traces actuelles sont toutes artificielles, tant au nord du baray oriental qu'à l'est d'Angkor Thom, ou encore plus au sud jusqu'à l'actuelle ville de Siemreap. Claude Jacques rapporte d'ailleurs «que [B. P. Groslier] lance apparemment comme une boutade : "à la limite je ne suis pas certain que le stung Siemreap existait" » (communication de B. P. Groslier non publiée, citée dans Jacques, $1978: 288$ ). En prenant à la lettre cette boutade, tout en ne la considérant que comme une hypothèse opératoire, plusieurs difficultés d'interprétation s'estompent alors que plusieurs autres vestiges s'organisent de manière cohérente.

Soulignons en particulier le cas de l'important canal bordé de digues CP505 qui file, parfaitement linéaire, depuis CP807 vers le sud et le lac. Cet ouvrage, dont l'existence n'a étonnamment pas été mentionnée par B. P. Groslier ${ }^{49}$, présente une direction qui relie l'angle sud-ouest de la douve d'Angkor Vat 414 au "débarcadère » occidental de Vat Athvéar 422, deux fondations attribuées à Sūryavarman II. Plus précisément, le canal n'apparaît qu'au sud de la digue extérieure de CP807, largement interrompue d'ailleurs à cet endroit. Aucune trace du canal n'apparaît en surface dans la " douve » de CP807. Les courbes de niveau et les reconnaissances permettent toutefois de suivre une dépression au nord de la digue intérieure, jusqu'à l'angle de la douve d'Angkor Vat, mais cette dépression n'a pas exactement la même orientation bien qu'elle soit dans le prolongement de CP505. D'autre part la brèche dans la digue intérieure de CP807 est particulièrement réduite par rapport à son pendant au sud ${ }^{50}$. Finalement, nos carottages (c10 à c16) indiquent que le canal CP505 se poursuit bien au nord de CP807 : à son emplacement présumé dans la douve de CP807 (c11 et c13), la stratigraphie présente justement une large plage de dépôts sableux de granulométrie parfois conséquente qui ne se retrouve pas dans les sondages voisins dans la "douve » $(\mathrm{c} 10 \text { et } \mathrm{c} 15)^{51}$. Il apparaît alors que ce canal CP505 a été interrompu par l'ouvrage CP807 et que ce dernier lui est donc postérieur.

48. On y note certaines ambiguïtés puisque les cours proposés suivent les contours d'ouvrages évidemment postérieurs (tel l'angle nord-ouest du baray oriental) et proviennent d'une capture qui peut dater de Rājendravarman.

49. L'ouvrage CP505 n'est pas mentionné dans le texte de la « Cité hydraulique ». Il n'apparaît que dans les cartes jointes à cet article, où il est considéré comme un des anciens cours présumés du stung Siemreap.

50. Le tronçon de la route Commaille est établi sur la berge occidentale de la dépression puis la traverse au nord de CP807 avant de couper la digue intérieure.

51. Le raccord du canal CP505 à Angkor Vat n'est cependant pas évident dans le carottage c12. Le détail de ce raccord dépasse toutefois le cadre de la présente étude. 
Au sud-ouest de Siemreap, la digue qui borde la rive droite du canal CP505 se perd $1400 \mathrm{~m}$ après avoir tranché un canal relié au baray occidental ${ }^{52}$, mais le canal CP505 se poursuit encore assez linéairement, au prix de quelques divagations, jusqu'à longer la face ouest du « débarcadère » de Vat Athvéar 422. Plus au sud, il rejoint encore un canal/digue CP572 qui gagne le pied oriental du Phnom Krom 423, puis le lac Tonle Sap. Si l'on considère les vestiges qui orientent ou bordent ce canal CP505, il apparaît que ce dernier peut être classé parmi les ouvrages de la première moitié du XII ${ }^{e}$ siècle. D'autre part, sa configuration présente du point de vue topographique une très nette analogie avec le canal CP574 qui relie le Bakong au lac et qui constituait vraisemblablement un chenal destiné à la navigation (B. P. Groslier, 1998 : 40 ; Pottier, 1999: 162) ${ }^{53}$. Il me semble que le canal CP505 a pu avoir la même fonction, et ainsi relier le lac à la capitale de la première moitié du XII ${ }^{\mathrm{e}}$ siècle ${ }^{54}$.

Avant de revenir à CP807, signalons encore qu'un second canal de même nature est situé non loin à l'est. Il s'agit du cours actuel de la rivière de Siemreap dans son tronçon qui, depuis les environs sud-est d'Angkor Vat, oblique vers le sud-ouest et traverse la ville de Siemreap. Divers éléments suggèrent que ce tronçon de rivière CP814, situé dans le prolongement du canal/digue CP572 qui rejoint le Phnom Krom 423, reprend le tracé d'un ancien canal réalisé au $\mathrm{X}^{\mathrm{e}}$ siècle pour relier les environs du baray oriental au lac Tonle Sap (Pottier, 1999 : 168 n. 514). Remarquons enfin au sud-est d'Angkor Vat que la digue CP806 présente une chicane lorsqu'elle franchit le canal CP814, indiquant par cette configuration particulière que même si la rivière est tardive, le canal CP814 est antérieur à cette digue CP806. La même chronologie relative peut d'ailleurs être avancée entre CP806 et la digue CP791 qui rejoint les environs orientaux du Phnom Bakheng au baray de Lolei à Rolûos.

Considérant maintenant l'ouvrage CP807 dans son contexte et dans une perspective hydraulique, nous devons remarquer, à la suite de B. P. Groslier, qu'il est branché à l'ouest sur l'angle du baray occidental et à l'est sur un angle formé par la digue qui descend au sud de l'angle sud-est d'Angkor Vat et par la digue CP806. Si aucun ouvrage de liaison «en dur » n'est actuellement visible à ces extrémités, l'absence de vestiges ne saurait en soi et sans recherches complémentaires contredire l'existence probable d'ouvrages régulateurs à date ancienne. Notons d'ailleurs que la digue orientale de l'extrémité sud-est de CP807 est interrompue par une brèche large d'une vingtaine de mètres à la base ${ }^{55}$. On pourrait envisager que CP807 a pu être utilisé afin de dériver une partie des eaux du baray vers la retenue que constitue la digue CP806 en bloquant le cours du canal CP814.

52. Ce canal CP504 prend naissance sur une trame de canaux CP483 située près de l'angle sudouest du baray occidental et plusieurs éléments suggèrent que ces ouvrages hydrauliques sont contemporains de ce baray (Pottier, 1999 : 119-123).

53. V. Goloubew a repéré en août 1932 l'embouchure du canal CP574 et l'a considérée comme "l'embarcadère de l'ancienne ville de Hariharālaya » (JF 9: 272). B. P. Groslier a d'ailleurs rapproché cet ouvrage du canal dit «de Damdek », situé à une trentaine de kilomètres à l'est d'Angkor, connu depuis le début du siècle pour avoir pu fournir une voie navigable privilégiée pour l'acheminement des blocs de grès depuis les carrières des Kulen.

54. Une telle fonction pour ce type d'ouvrage se heurte au scepticisme d'ingénieurs hydrauliciens qui y voient plutôt des ouvrages de drainage. Cette dernière fonction, que je reconnais bien volontiers, n'exclut a priori pas la première, d'autant que l'ancien régime hydraulique reste encore une inconnue de taille qui rend caduque toute comparaison avec les conditions actuelles.

55. Cette brèche n'est pas indiquée sur les plans topographiques de 1962 (Dumarçay et Pottier, 1993 : pl. 9), mais elle est reportée sur ceux de 1998 (JICA, $1998:$ pl. 9 au 1/5 000 et pl. 6 au 1/10 000 ). Elle est parfaitement sensible sur le terrain; la broussaille qui recouvre l'ensemble de la zone empêche cependant d'y noter de quelconques vestiges particuliers. 
Cependant, les niveaux à l'intérieur des deux digues montrent que le tronçon le plus bas de la «douve » est situé entre l'angle de CP807 et le canal CP505. Deux pentes convergent ainsi vers ce canal : la première depuis le baray occidental et la seconde depuis l'extrémité orientale de CP807. La brèche de l'extrémité orientale de CP807 peut alors être considérée comme une alimentation plutôt que comme un déversoir, ce qui renforce le lien étroit qui semble associer l'ouvrage CP807 au canal CP505 ${ }^{56}$. Soulignons enfin que, malgré l'état actuel d'altération des digues de CP807, l'examen des nivellements suggère qu'elles devaient à l'origine conserver sur toute leur longueur une altitude régulière, la digue intérieure aux environs de $23 \mathrm{~m}$ et l'extérieure vers $21 \mathrm{~m}$. En considérant une nappe d'eau à l'altitude de $20 \mathrm{~m}$, la contenance d'origine de CP807 peut être estimée à plus de 4,5 millions de $\mathrm{m}^{3}$ pour une surface de 125 ha.

Dans cette logique, les doubles digues de CP807 auraient constitué un chenal de dérivation, voire de stockage, d'une importante quantité d'eau provenant du baray occidental et, éventuellement, du baray oriental ou de ses environs via le blocage du canal CP814 ${ }^{57}$. Cette fonction serait plus en rapport avec la contenance des digues CP807 et expliquerait alors le plan et la hauteur de celles-ci en fonction des contraintes topographiques d'une telle dérivation en partie à contre-pente ${ }^{58}$. $\grave{A}$ l'appui de cette fonction, remarquons que le seul ouvrage voisin qui pourrait être comparé à CP807 a été interprété par $\mathrm{G}$. Trouvé comme un chenal d'alimentation ${ }^{59}$. La dérivation de CP807 peut donc avoir été destinée à subvenir, au moins temporairement, à l'alimentation du chenal CP505 dont j'ai indiqué plus haut qu'il pouvait constituer une voie navigable reliant la

56. Une histoire navrante est venue récemment confirmer que le point le plus bas de la « douve» correspond au canal CP505. En 1996, trois militaires hauts gradés habitant juste à l'est du Prasat Prei CP447 ont fait creuser un grand et profond bassin dûment agrémenté de fleurs et d'un bungalow (et revendu le remblai obtenu). Pour le mettre en eau, ils ont réalisé une tranchée le reliant au canal CP505 qui passe à proximité à l'ouest et ont bloqué ce canal par une digue haute de $2 \mathrm{~m}$ environ. Cela fut assez efficace pour remplir leur bassin. Mais dès les premières pluies, toutes les rizières de la "vallée artificielle » furent submergées jusqu'à la chaussée située dans l'axe occidental d'Angkor Vat. Tous les plants de riz furent perdus. Protégée par un blindé et une veille armée, cette digue fut l'objet d'un litige inique qui dura deux ans pendant lesquels aucune rizière ne put être cultivée en amont. Une buse a finalement été installée sous la digue, le bassin d'agrément est désormais à sec et les rizières cultivées. Il reste de cette triste anecdote qu'une simple digue de $2 \mathrm{~m}$ de haut située sur le canal CP505 à $400 \mathrm{~m}$ en aval de la brèche dans la digue extérieure de CP807 est à même d'inonder la majeure partie de la «vallée artificielle » sans autre source d'alimentation que pluviale.

57. Si cet angle a pu emmagasiner les eaux provenant des environs du baray oriental, sinon de celuici, les abords du sanctuaire sans nom CP870 durent alors être inondés. Le sanctuaire lui-même a pu rester hors eau. Remarquons d'ailleurs qu'il est construit sur un tertre nettement plus élevé, $4 \mathrm{~m}$ environ, que d'autres sanctuaires modestes de ce type.

58. Nous avons indiqué plus haut l'existence du Kôk Daun Bos CP654 à l'angle extérieur sud-ouest de CP807, près du point le plus bas des terrains extérieurs, à un emplacement permettant d'y envisager un exutoire éventuel. De fait, un canal traverse actuellement la digue extérieure, mais aucun élément ne nous a permis d'envisager que cette coupure soit d'origine.

59. Cet ouvrage repéré par G. Trouvé (1933: 1124, fig. 54 \& 55) est constitué de deux digues hautes de 4 à $6 \mathrm{~m}$ encadrant un espace large de $100 \mathrm{~m}$ environ. Il présente un trajet en « $\mathrm{L}$ » qui forme un angle au nord-ouest et se raccorde à l'angle nord-ouest de la douve d'Angkor Thom (cf. plan 5). On remarquera aussił'existence d'un ouvrage CP737 en partie comparable : il est situé à Rolûos et forme un U qui englobe les faces ouest et sud de Prei Monti 474, se prolonge vers l'est puis se retourne plein nord pour se raccorder à l'angle sud-est de la douve extérieure de Bakong 476. Il ne semble pas impossible qu'il soit lié au canal CP574 qui rejoint le lac, offrant ainsi une similitude tentante avec CP807. 
capitale au Tonle Sap ${ }^{60}$. La différence de hauteur des digues de CP807 et de CP505 ne permet cependant pas d'envisager que les embarcations aient pu mouiller dans la « douve ».

Enfin, plusieurs éléments suggèrent de repousser la datation de CP807 proposée par B. P. Groslier - la période d'Angkor Vat (B. P. Groslier, 1979: 183). Tout d'abord, il existe une légère mais sensible différence d'orientation entre la composition d'Angkor Vat et les digues de CP807 (respectivement $93^{\circ}$ et $91^{\circ}$ ). Si les raisons d'une telle différence nous échappent, on peut toutefois y voir une indication que ces deux ouvrages ne sont pas contemporains. D'autre part le canal CP505, que j'estime pouvoir dater de la première moitié du XII siècle, semble avoir été coupé par CP807, ce dernier ouvrage ayant pu être réalisé afin de suppléer un niveau d'eau insuffisant dans le canal. CP807 apparaît alors comme postérieur à la période d'Angkor Vat. Enfin, la similitude de CP807 avec le chenal d'alimentation repéré par G. Trouvé au nord d'Angkor Thom plaide aussi pour une date postérieure, car ce chenal donnant sur la douve d'Angkor Thom est au mieux contemporain de cette dernière. Il semble donc que l'ouvrage CP807 date au moins de la fin XII ${ }^{c}$, sinon du XIII siècle. Dans cette configuration, si Angkor Thom intra muros n'était pas directement accessible depuis le lac Tonle Sap, le canal CP505 permettait de s'en rapprocher à moins de $3 \mathrm{~km}$.

\section{L'extension de Yaśodharapura I}

Plusieurs types d'arguments s'accordent pour nier l'existence de Goloupura, cette vaste enceinte délimitant la capitale de Yaśovarman. Il semble donc que nous devions abandonner l'image d'une ville fermée dans une telle enceinte. Le cœur de cette Yaśodharapura I, tel qu'il a été mis en évidence par V. Goloubew puis par B. P. Groslier, reste cependant incontestable: le Phnom Bakheng 414, ses chaussées axiales et deux douves concentriques entre lesquelles se répartissent des sanctuaires satellites. Sans développer ici ce schéma relativement bien connu, mais en abandonnant l'hypothèse de V. Goloubew pour l'enceinte de la ville, nous pouvons désormais en guise de conclusion nous interroger sur la configuration, l'organisation et l'extension de cette capitale. Des recherches spécifiques et approfondies seraient nécessaires pour détailler cette question; cependant, les prospections et l'analyse des prises de vues aériennes offrent quelques éléments de réponse, ou plutôt quelques pistes de recherche.

Nous avons vu plus haut que B.P. Groslier souligne le rapprochement entre l'aménagement au pied du Phnom Bakheng et la configuration du Bakong (1979 : 174-175); ceci concerne, bien entendu, la composition des enceintes et de l'ensemble de temples satellites au pied du phnom. Mais à l'extérieur des enceintes du Bakheng, et à la différence de Bakong, un parcellaire a été généré par cette installation contemporaine et directement liée au baray oriental, le Yaśodharatatāka (Pottier, 1999 : 116-117). Nous avons souligné que ce dernier ouvrage présente à ses abords un parcellaire remarquablement isocline qui se poursuit jusqu'à englober le Phnom Bakheng : cet ensemble parcellaire n'est plus perceptible dans son intégralité, mais sa cohérence peut être suivie depuis les abords méridionaux du Yaśodharatatāka jusqu'au Phnom Bakheng. Si la trame de ce parcellaire reste inchangée dans l'ensemble, deux zones de densification parcellaire sont perceptibles: elles sont

60. Que ce canal puisse avoir été alimenté durant toute la période d'étiage reste une inconnue totale en l'absence de données précises sur les régimes pluviométriques et hydrologiques de l'époque. Notons par ailleurs que le chenal CP505 peut avoir aussi été alimenté par un étroit canal encadré de deux digues qui, $200 \mathrm{~m}$ au sud de la digue extérieure de CP807, vient de l'est et de l'angle constitué par la levée de terre prolongeant la face est d'Angkor Vat et par la digue CP806. Ce canal se poursuit (sans ses digues latérales) à l'ouest de CP505 et rejoint un canal CP811 provenant de la douve sud d'Angkor Thom près de son angle sud-ouest. Ce dernier canal est à l'évidence une réalisation postérieure aux digues CP807. 
localisées aux abords du Bakheng et de Pre Rup. En observant des clichés aériens de 1933, H. Marchal avait d'ailleurs été « frappé » par ce parcellaire très dense aux abords du Phnom Bakheng et de Pre Rup, deux sites ayant chacun pu correspondre au centre d'une capitale ; il y note une "mosaïque de petits rectangles plus ou moins orientés et qui représentent des rizières » et estime que " la présence de ces quadrilatères, rizières ou plantations, dénonce une partie habitée et par suite peut révéler des vestiges d'une ancienne ville » ( $R C A 02 / 34)$. Cette observation demeure particulièrement pertinente puisque l'analyse des prises de vues aériennes récentes de l'ensemble de la région sud d'Angkor confirme que ce parcellaire très dense, qui s'accompagne d'une concentration de terre-pleins de faibles dimensions, ne se trouve justement qu'à proximité directe du Phnom Bakheng et de Pre Rup ${ }^{61}$. Il permet ainsi de délimiter des zones de sur-concentration autour de ces fondations majeures. Si ce parcellaire et sa densité de terre-pleins ne sont pas suffisants à eux seuls pour préciser le détail d'un type d'habitat « urbain » (qui resterait au demeurant à préciser par des fouilles), ils témoignent au moins d'une occupation et d'aménagements spécifiques que l'on considérera comme caractéristiques de ces deux cités angkoriennes. Aussi peut-on interpréter l'extension du parcellaire isocline de densité moindre comme le témoin d'un développement directement lié à ces capitales.

Ce parcellaire particulier englobe le Phnom Bakheng et ses aménagements à la base, les chaussées et les bassins. Dans les zones exemptes de haute végétation, les prises de vues aériennes montrent que cette masse parcellaire s'étend, à l'ouest et au sud, sur une surface approximativement orthogonale dont les limites sont distantes d'environ $1500 \mathrm{~m}$ du centre du Phnom Bakheng ( $c f$. grisé clair sur plan 4). Cette répartition homogène pourrait rappeler, en plus réduite, l'image géométrique de Goloupura dont j'ai réfuté précédemment l'existence. Mais aucune marque linéaire ne détermine ici de limite franche autour de cette masse parcellaire. Par ailleurs, cette dernière s'étend aussi vers l'est, englobant par exemple le Prasat Kravan 388. Il ne semble donc s'agir que d'une «zone d'influence » de l'aménagement centré sur le Bakheng, plutôt que d'un parcellaire réalisé à l'intérieur d'une stricte délimitation éventuelle. Cet ensemble parcellaire présente toutefois une surface cohérente qui suggère une planification globalement géométrisée à partir d'éléments générateurs centraux. L'expérience de V. Goloubew a montré la fragilité d'une restitution d'un plan global à partir d'un simple tronçon conservé. Ici aussi, nous ne pouvons repérer que le détail du quart sud-ouest de la masse parcellaire qui englobait le temple-montagne de Yaśovarman et il est encore délicat de faire la part entre le parcellaire généré durant le règne de Yaśovarman et celui d'une "Yaśodharapura I bis » par Rājendravarman (Pottier, 1999: 196-197). Il serait donc ici aussi téméraire que vain d'en déduire un plan général sans autres données complémentaires.

Il reste pourtant que cette Yaśodharapura I peut être caractérisée par une composition ouverte, centrée sur le Bakheng, structurée par ses chaussées axiales et un parcellaire à l'orientation homogène. Trois anneaux schématiquement concentriques se distinguent : le complexe religieux au centre, une sur-concentration parcellaire accompagnée de terrepleins et de bassins, et en périphérie une extension parcellaire moins dense, se développant vers la face méridionale du Yaśodharatațāka où Yaśovarman fonda les ermitages des quatre sectes religieuses en activité sous son règne ${ }^{62}$.

Outre la géométrisation propre à la centralité du «temple-montagne », on observe donc une hiérarchisation de l'espace qui se traduit nettement d'un point de vue

61. Il importe toutefois de noter que les environs des temples pyramidaux postérieurs, pour la plupart, présentent une densité de végétation qui gêne le repérage du parcellaire fossile.

62. À propos des quatre ermitages de Yaśovarman, $c f$. Pottier, « About Yaśovarman's Buddhist āśrama in Angkor », Workshop on Buddhist Monastery, EFEO-SARC, Bangkok, 8-10 November 1999, à paraître. 
morphologique dans le parcellaire - à la différence de la capitale antérieure Hariharālaya. Par ailleurs, la rigueur et l'étendue du parcellaire de Yaśodharapura I témoignent d'une évidente volonté de maîtrise du territoire sur lequel s'implante la nouvelle capitale. L'établissement d'une trame parcellaire homogène générée par les deux éléments majeurs du schéma urbain, le Bakheng et son baray, apparaît alors comme un moyen privilégié pour remodeler l'environnement et ainsi engager, hors les murs du temple central, une réelle mise en conformité du territoire pour "mettre en ordre l'univers ${ }^{63}$. La rupture apparaît alors encore plus nettement avec l'ancienne capitale si l'on considère que Yaśodharapura I, s'appuyant sur le développement de nouveaux aménagements hydrauliques, quitte les rives naturellement inondées du Tonle Sap pour les terres en amont. Cette conquête de l'arrière-pays se poursuit d'ailleurs à l'extrême avec le déplacement éphémère de la capitale à Koh Ker en 921 par Jayavarman IV.

Le retour de Rājendravarman à Angkor au milieu du $X^{e}$ siècle nous ramène enfin à l'une des répercussions principales de l'abandon de l'hypothèse de Goloupura. Hariharālaya, Yaśodharapura I et Chok Gargyar (Koh Ker) offrent alors une succession de cités ouvertes, sans enceinte, qui souligne le caractère fondamentalement centrifuge de ces capitales et permet de reconsidérer différemment - peut-être plus souplement - leurs superpositions et leurs glissements durant les deux siècles d'occupation qui séparent « Yaśodharapura I bis » de l'apparition d'Angkor Thom sous le règne de Jayavarman VII. Dans le cas de la capitale de Rājendravarman par exemple, l'analyse des vestiges et la télédétection permettent de dégager les premiers éléments d'un schéma urbain centré sur Pre Rup (Pottier, 1999 : 176-199). La composition ouverte de cette capitale baptisée par P. Stern «ville de l'Est» (1954: 678) se situe alors dans la tradition des capitales antérieures et poursuit le développement de l'aménagement angkorien. À l'opposé, fortement délimitée par son enceinte et sa large douve, Angkor Thom n'apparaît plus guère comme un représentant fidèle de l'urbanisme angkorien. Elle constitue plutôt une rupture brutale qui sonne le glas des déplacements de la capitale, et un achèvement en se fixant dans son enceinte pour plus de deux siècles. Si elle reprend des principes employés dans les cités précédentes, elle invente une nouvelle forme urbaine fermée probablement liée à l'apprentissage dramatique, en 1177, qu'Angkor n'est plus invincible. Cette période marque donc l'introduction de systèmes défensifs massifs au cœur même du royaume khmer. Mais Angkor Thom signale aussi - au moins formellement - le début d'une dissociation entre la cité et son territoire, deux composantes auparavant étroitement liées l'une à l'autre dans un remarquable continuum urbain-rural. Les aménagements territoriaux particulièrement nombreux sous le règne de Jayavarman VII suggèrent toutefois que cette séparation dut être progressive. En ce sens, il est significatif que faisant suite à deux siècles d'urbanisme ouvert, le projet de Jayavarman VII se soit attaché à établir au centre de la nouvelle capitale enclose un «temple-montagne», le Bayon, qui constitue un véritable microcosme du royaume.

63. Sur la «mise en ordre de l'univers » par l'architecture, voir Dumarçay, 1998 : 79-86. L'aménagement du territoire se traduit aussi à cette époque par le développement de grandes fondations privées établies autour de vastes trapéang en périphérie angkorienne, d'établissements particuliers aux phnom environnants ou encore par l'apparition de chaussées qui relient des points éloignés du territoire, voire du royaume (Pottier, $1999: 169-175$ ). 


\section{Bibliographie}

BOISSELIER, Jean

1966 Asie du Sud-Est, I: Le Cambodge, Paris, Picard (Manuel d'archéologie d'Extrême-Orient, 1).

CÆDÈs, George

1911 «Une nouvelle inscription du Phnom Bàkhen », BEFEO, 11/1, [rééd. 1989, Articles sur le pays khmer, tome I, Paris, EFEO (Réimpressions), p. 6-8].

1952 Inscriptions du Cambodge, vol. 4, Paris, E. de Boccard (Collection des textes et documents sur l'Indochine, 3 ).

1964a Les états hindouisés d'Indochine et d'Indonésie, [rééd. 1989, Paris, E. de Boccard], Histoire du Monde, vol. VII, Paris.

1964b Inscriptions du Cambodge, vol. 7, Paris, EFEO (Collection des textes et documents sur l'Indochine, 3).

CEDÈs, George, et DuPONT, Pierre

1943-46 «Les stèles de Sdǒk Kăk Thom, Phnom Sandak et Práh Vihãr «, BEFEO, 43, p. 56-154, [rééd. 1992, Articles sur le pays khmer, tome II, Paris, EFEO (Réimpressions), p. 167-265].

DUMARÇAY, Jacques

1998 L'architecture et ses modèles en Asie du Sud-Est, Paris, Librairie Oriens.

DUMARÇAY, Jacques et Groslier, Bernard Philippe

1973 Le Bayon. Histoire architecturale du temple. Inscriptions du Bayon, 2 vol., Paris, EFEO (Mémoires archéologiques, 3).

DuMARÇAY, Jacques, (introd.) et POTTIER, Christophe, (préparé par)

1993 Documents topographiques de la Conservation des monuments d'Angkor, Paris, EFEO (Mémoires archéologiques, 21).

FINOT, Louis

1925 «Inscriptions d'An்kor », BEFEO, 25/3-4, p. 289-409.

GARAMI, Ferenc et KERTAL, Istvan

1993 Water Management in the Angkor Area, Budapest, Angkor Foundation.

GAUCHER, Jacques

1996 Études archéologiques à Angkor: de Yasodharapura à Angkor Thom, EFEO, dactyl., Paris.

1997 Études archéologiques à Angkor : de Yasodharapura à Angkor Thom, Rapport campagne 1996-1997, ministère des Affaires étrangères, dactyl., Paris.

GLAIZE, Maurice

1963 Les monuments du groupe d'Angkor, $3^{\mathrm{e}}$ éd., Paris, Adrien Maisonneuve, [4 ${ }^{\mathrm{c}}$ éd. revue et mise à jour par Jean Boisselier, 1993].

GOLOUBEW, Victor

1933a «Le Phnom Bàkheñ et la ville de Yaśovarman. Rapport sur une mission archéologique dans la région d'An̉kor en août-novembre 1932 », BEFEO, 33/1, p. 319-344.

1933 b «Chronique. Yaçodharapura I », BEFEO, 33/2, p. 1119-1120.

1934 «Nouvelles recherches autour du Phnom Bàkhen. Rapport sur une mission archéologique dans la région d'Añkor (décembre 1933 - mars 34) », BEFEO, 34/2, p. 576-600.

1936 «Mission Goloubew, recherches dans Anikor Thom », BEFEO, 36/2, p. 619-623.

1937 «Recherches dans Anikor Thom », BEFEO, 37/2, p. 651-655. 
GROSLIER, Bernard Philippe

1961 Indochine. Carrefour des Arts, Paris, Albin Michel, [rééd. 1983].

1965 Découvertes archéologiques récentes au Cambodge, Siemreap, dactyl., 10 p.

1979 «La cité hydraulique angkorienne: exploitation ou surexploitation du sol ? », BEFEO, 66, p. 161-202.

1998 Mélanges sur l'Archéologie du Cambodge, textes réunis par Jacques Dumarçay, Paris, EFEO (Réimpressions, 10).

Higham, Charles

1989 The Archaeology of Mainland Southeast Asia, Cambridge, Cambridge University Press.

ISHIZAWA, Yoshiaki et TAMURA, Hitoshi

1999 Along the royal roads to Angkor, New York/Tôkyô, Weatherhill.

JACQUES, Claude

1970 «Études d'épigraphie cambodgienne - 4. Deux inscriptions du Phnom Bàkhen (K. 464 et K. 558) - 5. La stèle du Prasat Cha Chuk (K. 1034) », $B E F E O, 57$, p. 57-89.

1978 «Études d'épigraphie cambodgienne - 10. Autour de quelques toponymes de l'inscription de Pràsàt Trapãn Run K. 598. La capitale angkorienne de Yaśovarman $\mathrm{I}^{\text {er }}$ à Sūryavarman $\mathrm{I}^{\mathrm{cr}} », B E F E O, 65 / 1$, p. 281-321.

1990 Angkor, Paris, Bordas.

JICA (Japan International Cooperation Agency)

1998 Topographic Map, $5000^{\circ} \& 10000^{\circ}$, Tôkyô, JICA, 10 \& 12 feuilles.

MARCHAL, Henri

1936 «Chronique. Recherches dans Anjkor Thom », BEFEO, 36/2, p. 619-623.

1937 «Chronique. Nivellement partiel d'Ankor Thom», BEFEO, 37/2, p. 656664.

PARMENTIER, Henri

1933 «Examen du levé d'avion du parc d'Añkor par le Capitaine Gouët les 24-26 janvier 1933 à 9 heures à l'altitude de $1300 \mathrm{~m} », B E F E O, 33 / 2$, Hanoi, p. 1111-1116.

POTTIER, Christophe

1999 Carte archéologique de la région d'Angkor - Zone sud, Université de Paris III, Thèse de Doctorat dactylographiée, Bruno Dagens dir., Paris, 3 vol. (41 cartes).

STERN, Philippe

1927 Le Bayon d'Angkor Thom et l'évolution de l'art khmer, Paris, Paul Geuthner, (Annales du musée Guimet, Bibliothèque de vulgarisation, tome 47).

1954 «Diversité et rythme des fondations royales khmères », BEFEO, 47/2, p. 649687.

TROUVE, Georges

1933 «Chronique. Chaussées et canaux autour d'Annkor Thom », BEFEO, 33/2, p. $1120-1128$. 